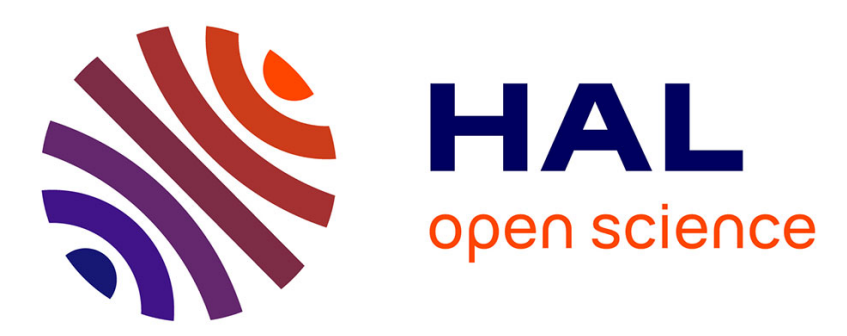

\title{
Dynamically Scaled Immersion and Invariance Adaptive Control for Euler-Lagrange Mechanical Systems
}

\author{
Sungpil Yang, Maruthi Akella, Frédéric Mazenc
}

\section{To cite this version:}

Sungpil Yang, Maruthi Akella, Frédéric Mazenc. Dynamically Scaled Immersion and Invariance Adaptive Control for Euler-Lagrange Mechanical Systems. Journal of Guidance, Control, and Dynamics, 2017, 40 (11), pp.2844-2856. 10.2514/1.G002177 . hal-01662280

\section{HAL Id: hal-01662280 \\ https://hal.inria.fr/hal-01662280}

Submitted on 13 Dec 2017

HAL is a multi-disciplinary open access archive for the deposit and dissemination of scientific research documents, whether they are published or not. The documents may come from teaching and research institutions in France or abroad, or from public or private research centers.
L'archive ouverte pluridisciplinaire HAL, est destinée au dépôt et à la diffusion de documents scientifiques de niveau recherche, publiés ou non, émanant des établissements d'enseignement et de recherche français ou étrangers, des laboratoires publics ou privés. 


\title{
Dynamically Scaled Immersion and Invariance Adaptive Control for Euler-Lagrange Mechanical Systems
}

\author{
Sungpil Yang* and Maruthi R. Akella ${ }^{\dagger}$ \\ University of Texas at Austin, Austin, Texas, 78712 \\ Frédéric Mazenc \\ EPI DISCO Inria, 91192, Gif-sur-Yvette, France
}


This paper addresses adaptive control of specific Euler-Lagrange systems: rigidbody attitude control, and the n-link robot manipulator. For each problem, the model parameters are unknown but the lower bound of the smallest eigenvalue of the inertia matrix is assumed to be known. The dynamic scaling Immersion and Invariance (I\&I) adaptive controller is proposed to stabilize the system without employing a filter for the regressor matrix. A scalar scaling factor is instead implemented to overcome the integrability obstacle that arises in I\&I adaptive control design. First, a filter-free controller is proposed for the attitude problem such that the rate feedback gain is proportional to the square of the scaling factor in the tracking error dynamics. The gain is then shown to be bounded through state feedback while achieving stabilization of the tracking error. The dynamic scaling factor increases monotonically by design and may end up at a finite but arbitrarily large value. However, by introducing three more dynamic equations, the non-decreasing scaling factor can be removed from the closed-loop system. Moreover, the behavior of dynamic gain is dictated by design parameters so that its upper bound is limited by a known quantity and its final value approaches the initial value. A similar approach for the dynamic gain design is also applied to a filter-dependent controller where a filter for the angular rate is utilized to build a parameter estimator. Unlike the filter-free design, the filter-dependent controller admits a constant gain for the rate feedback while the dynamic scaling factor rather appears in the filter. Finally, the proposed design is applied to robot manipulator systems. Spacecraft attitude and 2-link planar robot tracking problems are considered to demonstrate the performance of the controllers through simulations.

\footnotetext{
${ }^{*}$ Ph.D. Candidate, Department of Aerospace Engineering and Engineering Mechanics, 1 University Station; yang.sungpil@utexas.edu. Student Member AIAA

†Professor, Department of Aerospace Engineering and Engineering Mechanics, 1 University Station. Myron L. Begeman Fellow in Engineering; makella@mail.utexas.edu. Associate Fellow AIAA

${ }^{\ddagger}$ Chargé de Recherche, EPI DISCO Inria, Laboratoire des Signaux et Systèmes (L2S, UMR CNRS 8506), CNRS, CentraleSupélec, Université Paris-Sud, 3 rue Joliot Curie; frederic . mazenc@ l2s . centralesupelec . fr.
} 


\section{Introduction}

In most nonlinear control problems, designing a satisfactory controller to force system states to track prescribed reference trajectories usually requires model parameters. Closed-loop performance depends largely on how well the mathematical model and the physical plant match. To improve the quality of the controllers, system identification can be performed to improve plant model before controller design, but it is not always perfect. Adaptive control has been extensively studied as a way of dealing with parameter uncertainties $[1,2]$. The philosophy of adaptive control is to control dynamic systems using system parameters estimated online so that robustness to parameter uncertainties automatically follows.

Classical adaptive control design obeys the so-called Certainty Equivalence (CE) principle. First, a controller is designed with system parameters assumed to be known. Then the parameters are replaced by estimated values. A parameter update law is determined through the judicious use of a Lyapunov(-like) function such that a tracking objective is fulfilled. The estimate errors remain bounded as the error terms in the time derivative of the Lyapunov(-like) function are canceled by the update law. Departing from the CE philosophy, a new methodology called Immersion and Invariance (I\&I) adaptive control was recently proposed promising better performance and flexibility of parameterization structure $[3,4]$. Unlike CE, the estimates do not come directly from the update law. Instead, a function which satisfies a certain partial differential equation (PDE) is combined with the states of the update law to form the estimates. The norm of the estimation error monotonically decreases, and thus, the estimated parameters approach the true values as time evolves-a feature not observed in CE-based controllers. Also, the resulting estimate error dynamics allow us to dominate the perturbation term in the Lyapunov(-like) function derivative without the fragile cancellation operation. This new methodology allows non-affine parameterization for some applications and has been shown to outperform CE-based controllers in transient performance $[5-11]$.

When the I\&I method was first proposed, applications were limited because designing an I\&I adaptive controller typically leads to solving a partial differential equation (PDE) $[3,4]$. The solution to the PDE always exists for autonomous single-input systems. Existence of solutions to such 
PDEs is not guaranteed for general multi-input systems-a condition commonly known as the "integrability obstacle". However, by introducing low-pass filters for the state and regressor matrix, this obstacle is shown to be circumvented. Various I\&I adaptive controllers have been proposed for linear and nonlinear systems as a result $[5,8,11]$.

Recently, an alternate method to avoid the integrability obstacle was proposed in [12]. Aided by a state filter with a dynamic scaling factor appearing in the gain, the PDE becomes solvable. A scaled manifold is proven to be globally stable via a certain choice of the scaling factor dynamics. In [13] and [14], the dynamic scaling method is applied to aircraft flight control systems. In the special case where the system is monotonizable and parameters have known bounds, it is shown that the parameter estimates converge to the true values exponentially fast and the scaling factor returns to unity as the tracking error goes to zero [15]. Compared to the existing method that requires both state and regressor filters, this approach achieves similar performance as the lowerdimensional dynamic extensions.

It is possible to bring the dynamic gain down to the initial value when the dynamic scaling approach is used taking advantage of the structural properties like observability [12]. However, in most adaptive control design cases the scaling factor does not have a convergent term in its dynamics and grows monotonically to some unknown constant. This feature can be a drawback because we do not know how large the scaling factor becomes, although it is finite, or whether it can be reset. This could result in "high-gain" control action and cause undesirable transient behavior of the closed-loop system.

In the literature, many model reference adaptive controllers have been developed for the spacecraft attitude control and robot manipulator systems as their dynamic structures admit affineparameterization for the inertia parameters [16-21]. Unfortunately, ideal I\&I adaptive controllers do not exist as no solutions to the PDE of these systems exist. This is due to the nonlinear gyroscopic term in the momentum-level equations of motion. Only the filter approach was applied to the attitude control and robot manipulator systems respectively in [9] and [10] to overcome the integrability obstacle. In this work, we apply the dynamic scaling method to the quaternion-based attitude tracking control problem. Also, we modify the dynamic scaling factor so that it does not 
increase monotonically but instead converges to the vicinity of the initial value by introducing three scalar dynamics. Moreover, based on the local Lipschitz condition of the regressor matrix, a filter-free controller is proposed that achieves even lower dimensional dynamic extensions. Due to the structural analogy between the attitude and Euler-Lagrange dynamics, the proposed design scheme is also applied to $n$-link robot manipulator systems. The preliminary works are reported in [22-24]. The performances of the tracking controllers for both attitude and 2-link planar robot arm dynamics are demonstrated and compared through numerical simulations.

This paper is organized as follows: The governing equations of a rigid-body rotational motion are described in Section II. Both filter-free and filter-dependent controllers are proposed with stability proofs in Section III. In Section IV, the proposed design methods are shown to be applicable to a subset of Euler-Lagrange mechanical systems covering $n$-link robot manipulators. Numerical simulations of spacecraft attitude and 2-link planar robot controllers are carried out in Section V. Finally, concluding remarks are presented in Section VI.

\section{Model Description}

\section{A. Dynamic Equations}

Rigid-spacecraft attitude motion is governed by Euler's rotational dynamics. Let $J \in \mathbb{R}^{3 \times 3}$ be a positive definite matrix that represents the inertia of a spacecraft. In the body-fixed frame of the spacecraft, let $\omega(t) \in \mathbb{R}^{3}$ and $\mathbf{u} \in \mathbb{R}^{3}$ be the angular rate and the external control torque respectively. Then the governing equation is described as

$$
J \dot{\omega}(t)=-S(\omega(t)) J \omega(t)+\mathbf{u}(t),
$$

where $S: \mathbb{R}^{3} \rightarrow \mathbb{R}^{3 \times 3}$ is the skew-symmetric matrix operator such that $S(\mathbf{v}) \mathbf{w}=\mathbf{v} \times \mathbf{w}$ for any $\mathbf{v}, \mathbf{w} \in \mathbb{R}^{3}$. We adopt the quaternion vector

$$
\mathbf{q}(t) \in \mathbb{Q}=\left\{\mathbf{q}=\left(q_{0}, \mathbf{q}_{v}\right) \in \mathbb{R} \times \mathbb{R}^{3} \mid \mathbf{q}^{T} \mathbf{q}=1\right\}
$$


to represent the body attitude, the orientation of the body-fix frame $\mathcal{F}_{B}$ with respect to the inertial frame $\mathcal{F}_{I}$. The corresponding kinematics are then given by

$$
\dot{\mathbf{q}}(t)=\frac{1}{2} E(\mathbf{q}(t)) \omega(t)
$$

with the function $E: \mathbb{Q} \rightarrow \mathbb{R}^{4 \times 3}$ defined as

$$
E(\mathbf{q})=\left[\begin{array}{c}
-\mathbf{q}_{v}^{T} \\
S\left(\mathbf{q}_{v}\right)+q_{0} I_{3}
\end{array}\right],
$$

where $I_{3} \in \mathbb{R}^{3 \times 3}$ is the identity matrix. Note that the corresponding direction cosine matrix is obtained from the identity [25]

$$
C(\mathbf{q})=I_{3}-2 q_{0} S\left(\mathbf{q}_{v}\right)+2 S^{2}\left(\mathbf{q}_{v}\right)
$$

Let the reference quaternion $\mathbf{q}_{r}(t)$ represent the rotation of the reference frame $\mathcal{F}_{R}$ from the inertial frame $\mathcal{F}_{I}$ and the reference rate $\omega_{r}(t)$ be described in the frame $\mathcal{F}_{R}$. Then the tracking errors $\delta \mathbf{q}(t) \in \mathbb{Q}$ and $\delta \omega(t) \in \mathbb{R}^{3}$ are defined as [9]

$$
\begin{aligned}
C(\delta \mathbf{q}(t)) & =C(\mathbf{q}(t)) C^{T}\left(\mathbf{q}_{r}(t)\right), \\
\delta \omega(t) & =\omega(t)-\Omega(t),
\end{aligned}
$$

where $\Omega(t)=C(\delta \mathbf{q}(t)) \omega_{r}(t)$ is the reference rate in the body-fixed frame $\mathcal{F}_{B}$. Using the kinematic equation for the rotation matrix

$$
\frac{d}{d t} C(\mathbf{q}(t))=-S(\omega(t)) C(\mathbf{q}(t))
$$


and the matching conditions

$$
\begin{aligned}
\dot{\mathbf{q}}_{r}(t) & =\frac{1}{2} E\left(\mathbf{q}_{r}(t)\right) \boldsymbol{\omega}_{r}(t) \\
\dot{\omega}_{r}(t) & =\mathbf{f}(t),
\end{aligned}
$$

where the forcing term $\mathbf{f}(t) \in \mathbb{R}^{3}$ is assumed to be a predefined function such that $\omega_{r}, \mathbf{f}, \dot{\mathbf{f}} \in L_{\infty}$, the error dynamics are obtained as [9]

$$
\begin{aligned}
\delta \dot{\mathbf{q}}(t) & =\frac{1}{2} E(\delta \mathbf{q}(t)) \delta \omega(t) \\
J \delta \dot{\omega}(t) & =-S(\omega(t)) J \omega(t)+\mathbf{u}(t)+J(S(\omega(t)) \Omega(t)-C(\delta \mathbf{q}(t)) \mathbf{f}(t)) .
\end{aligned}
$$

\section{B. Parameterization}

Let us define the model parameter vector $\boldsymbol{\theta}^{*}=\left(\theta_{1}, \theta_{2}, \theta_{3}, \theta_{4}, \theta_{5}, \theta_{6}\right) \in \mathbb{R}^{6}$ obtained from the inertia matrix

$$
J=\left[\begin{array}{lll}
\theta_{1} & \theta_{6} & \theta_{5} \\
\theta_{6} & \theta_{2} & \theta_{4} \\
\theta_{5} & \theta_{4} & \theta_{3}
\end{array}\right] .
$$

The parameters are assumed to be unknown but the lower bound of the smallest eigenvalue of $J$ denoted as $J_{m}$ is to be known. It is known for any $\mathbf{x}, \omega, \Omega \in \mathbb{R}^{3}$ that the regressor matrix $\Phi(\mathbf{x}, \omega, \Omega)$ is defined such that

$$
J \mathbf{x}-S(\omega) J \omega+J S(\omega) \Omega=\Phi(\mathbf{x}, \omega, \Omega) \boldsymbol{\theta}^{*}
$$

Also, $\Phi(\mathbf{x}, \omega, \Omega)$ is decomposed as

$$
\Phi(\mathbf{x}, \omega, \Omega)=\Phi_{1}(\mathbf{x})+\Phi_{2}(\omega, \Omega)
$$


such that $\Phi_{1}(\mathbf{x}) \boldsymbol{\theta}^{*}=J \mathbf{x}$ and $\Phi_{2}(\omega, \Omega) \boldsymbol{\theta}^{*}=-S(\omega) J \boldsymbol{\omega}+J S(\omega) \Omega$. For $\mathbf{x}=\left(x_{1}, x_{2}, x_{3}\right), \boldsymbol{\omega}=$ $\left(\omega_{1}, \omega_{2}, \omega_{3}\right)$ and $\Omega=\left(\Omega_{1}, \Omega_{2}, \Omega_{3}\right)$, the explicit forms of $\Phi_{1}(\mathbf{x})$ and $\Phi_{2}(\omega, \Omega)$ are given by

$$
\Phi_{1}(\mathbf{x})=\left[\begin{array}{cccccc}
x_{1} & 0 & 0 & 0 & x_{3} & x_{2} \\
0 & x_{2} & 0 & x_{3} & 0 & x_{1} \\
0 & 0 & x_{3} & x_{2} & x_{1} & 0
\end{array}\right]
$$

and

$$
\Phi_{2}(\omega, \Omega)=\left[\begin{array}{ccc}
\omega_{2} \Omega_{3}-\omega_{3} \Omega_{2} & -\omega_{3} \omega_{1} & \omega_{1} \omega_{2} \\
\omega_{2} \omega_{3} & \omega_{3} \Omega_{1}-\omega_{1} \Omega_{3} & -\omega_{1} \omega_{2} \\
-\omega_{2} \omega_{3} & \omega_{3} \omega_{1} & \omega_{1} \Omega_{2}-\omega_{2} \Omega_{1} \\
\omega_{3}^{2}-\omega_{2}^{2} & \omega_{1} \Omega_{2}-\omega_{2} \Omega_{1}+\omega_{1} \omega_{2} & \omega_{3} \Omega_{1}-\omega_{1} \Omega_{3}-\omega_{3} \omega_{1} \\
\omega_{1} \Omega_{2}-\omega_{2} \Omega_{1}-\omega_{1} \omega_{2} & \omega_{1}^{2}-\omega_{3}^{2} & \omega_{2} \Omega_{3}-\omega_{3} \Omega_{2}+\omega_{2} \omega_{3} \\
\omega_{3} \Omega_{1}-\omega_{1} \Omega_{3}+\omega_{3} \omega_{1} & \omega_{2} \Omega_{3}-\omega_{3} \Omega_{2}-\omega_{2} \omega_{3} & \omega_{2}^{2}-\omega_{1}^{2}
\end{array}\right]^{T}
$$

Once the target dynamics are set to

$$
\delta \dot{\omega}(t)=-k_{p} \delta \mathbf{q}_{v}(t)-k_{v}(t) \delta \omega(t)
$$

where $k_{p}>0$ is the constant scalar gain for the vector part of the error quaternion and $k_{v}(t)>0$ is the scalar gain for the rate error, which can be static or dynamic depending on parameter estimator design, Eq. (10) is rewritten as

$$
\begin{aligned}
\delta \dot{\omega} & =-k_{p} \delta \mathbf{q}_{v}-k_{v} \delta \omega+J^{-1}\left[\mathbf{u}+J\left(k_{p} \delta \mathbf{q}_{v}+k_{v} \delta \omega-C(\delta \mathbf{q}) \mathbf{f}\right)-S(\omega) J \omega+J S(\omega) \Omega\right] \\
& =-k_{p} \delta \mathbf{q}_{v}-k_{v} \delta \omega+J^{-1}\left(\mathbf{u}+\Phi(\mathbf{x}, \omega, \Omega) \boldsymbol{\theta}^{*}\right)
\end{aligned}
$$

where the vector $\mathbf{x}$ is specifically defined here as

$$
\mathbf{x}=k_{p} \delta \mathbf{q}_{v}+k_{v} \delta \omega-C(\delta \mathbf{q}) \mathbf{f}
$$


In the above equations, the time argument for the states and control is omitted for notational simplicity. From here on, function arguments are often omitted whenever no confusion can arise in the context.

The objective is to design I\&I adaptive controllers which achieve $\left(\delta \mathbf{q}_{v}, \delta \boldsymbol{\omega}\right) \rightarrow(\mathbf{0 , 0})$ as $t \rightarrow \infty$ while estimating the parameter $\boldsymbol{\theta}^{*}$.

\section{I\&I Adaptive Controller Design}

In this section, two types of I\&I adaptive controllers are proposed based on the dynamic scaling method. We first consider the filter-free controller where the scaling factor appears in the feedback gain $k_{v}(t)$ so the control effort is directly affected by the dynamic gain. When the gain $k_{v}(t)$ is desired to be static, we can introduce a filter for the state $\omega(t)$ so that the dynamic scaling factor is implemented only in the filter. In the second case, we fix the rate feedback gain $k_{v}(t)=k_{v}$ and apply the design procedure proposed in [12] in a slightly different way.

The control law for the both cases is proposed as

$$
\mathbf{u}(t)=-\Phi(\mathbf{x}(t), \omega(t), \Omega(t))(\hat{\boldsymbol{\theta}}(t)+\boldsymbol{\beta}(t)),
$$

where $(\hat{\boldsymbol{\theta}}+\boldsymbol{\beta}) \in \mathbb{R}^{6}$ is the estimate for $\boldsymbol{\theta}^{*}$ to be determined. So the closed-loop rate dynamics become

$$
\delta \dot{\omega}(t)=-k_{p} \delta \mathbf{q}_{v}(t)-k_{v}(t) \delta \omega(t)-J^{-1} \Phi(\mathbf{x}(t), \omega(t), \Omega(t)) \tilde{\boldsymbol{\theta}}(t),
$$

where $\tilde{\boldsymbol{\theta}}(t)=\hat{\boldsymbol{\theta}}(t)+\boldsymbol{\beta}(t)-\boldsymbol{\theta}^{*}$.

Before estimator design, the common blocks for both methods are built in the following. Let

$$
\mathbf{y}=k_{p} \delta \mathbf{q}_{v}-k_{v} \Omega-C(\delta \mathbf{q}) \mathbf{f}
$$


Then by linearity of $\Phi_{1}$, we have

$$
\begin{aligned}
\Phi_{1}(\mathbf{x}) & =\Phi_{1}\left(\mathbf{y}+k_{v} \omega\right) \\
& =\Phi_{1}(\mathbf{y})+k_{v} \Phi_{1}(\omega)
\end{aligned}
$$

Since, for all $i, j \in\{1,2,3\}$,

$$
\frac{\partial \boldsymbol{\phi}_{1 i}}{\partial \omega_{j}}=\frac{\partial \boldsymbol{\phi}_{1 j}}{\partial \omega_{i}},
$$

where $\phi_{1 i}$ and $\phi_{1 j}$ are the $i$ th and $j$ th columns of $\Phi_{1}^{T}(\mathbf{x})$ respectively, there is $\mu_{1}$ such that

$$
\frac{\partial \mu_{1}}{\partial \omega}=\Phi_{1}^{T}(\mathbf{x})
$$

Let

$$
\boldsymbol{\mu}_{1}^{*}(\boldsymbol{\omega})=\left[\begin{array}{llllll}
\frac{1}{2} \omega_{1} & \frac{1}{2} \omega_{2} & \frac{1}{2} \omega_{3} & \omega_{2} \omega_{3} & \omega_{3} \omega_{1} & \omega_{1} \omega_{2}
\end{array}\right]^{T}
$$

Then a solution to Eq. (24) is given by

$$
\mu_{1}=\Phi_{1}^{T}(\mathbf{y}) \omega+k_{v} \mu_{1}^{*}(\omega)
$$

However, for $\Phi_{2}(\omega, \Omega)$, there is no $\mu_{2}$ satisfying

$$
\frac{\partial \mu_{2}}{\partial \omega}=\Phi_{2}^{T}(\omega, \Omega)
$$

because

$$
\frac{\partial \boldsymbol{\phi}_{2 i}}{\partial \omega_{j}} \neq \frac{\partial \boldsymbol{\phi}_{2 j}}{\partial \omega_{i}}
$$

for all $i, j \in\{1,2,3\}$, where $\phi_{2 i}$ and $\phi_{2 j}$ are the $i$ th and $j$ th columns of $\Phi_{2}^{T}(\omega, \Omega)$. This is known as the integrability obstacle and is the reason why the I\&I adaptive control design method cannot be directly applied to the rigid-body attitude control problem [3]. So we consider a solvable PDE by 
introducing a function $\Psi(\omega, \Omega)$ satisfying

$$
\frac{\partial \boldsymbol{\phi}_{2_{i}}}{\partial \omega_{j}}+\frac{\partial \boldsymbol{\psi}_{i}}{\partial \omega_{j}}=\frac{\partial \boldsymbol{\phi}_{2_{j}}}{\partial \omega_{i}}+\frac{\partial \boldsymbol{\psi}_{j}}{\partial \omega_{i}}
$$

for all $i, j \in\{1,2,3\}$, where the subscripts $i$ and $j$ for $\psi$ indicate the $i$ th and $j$ th columns of the matrix $\Psi^{T}$, i.e., there is a solution to the PDE

$$
\frac{\partial \mu_{2}}{\partial \omega}=\Phi_{2}^{T}(\omega, \Omega)+\Psi^{T}(\omega, \Omega)
$$

The particular choice

$$
\boldsymbol{\mu}_{2}(\boldsymbol{\omega}, \Omega)=\left[\begin{array}{c}
\omega_{1}\left(\omega_{2} \Omega_{3}-\omega_{3} \Omega_{2}\right) \\
\omega_{2}\left(\omega_{3} \Omega_{1}-\omega_{1} \Omega_{3}\right) \\
\omega_{3}\left(\omega_{1} \Omega_{2}-\omega_{2} \Omega_{1}\right) \\
\left(\omega_{3}^{2}-\omega_{2}^{2}\right)\left(\omega_{1}+\frac{1}{2} \Omega_{1}\right) \\
\left(\omega_{1}^{2}-\omega_{3}^{2}\right)\left(\omega_{2}+\frac{1}{2} \Omega_{2}\right) \\
\left(\omega_{2}^{2}-\omega_{1}^{2}\right)\left(\omega_{3}+\frac{1}{2} \Omega_{3}\right)
\end{array}\right]
$$

allows us to have

$$
\Psi(\omega, \Omega)=\left[\begin{array}{ccc}
0 & \omega_{1}\left(\omega_{3}+\Omega_{3}\right) & -\omega_{1}\left(\omega_{2}+\Omega_{2}\right) \\
-\omega_{2}\left(\omega_{3}+\Omega_{3}\right) & 0 & \omega_{2}\left(\omega_{1}+\Omega_{1}\right) \\
\omega_{3}\left(\omega_{2}+\Omega_{2}\right) & \omega_{3}\left(\omega_{1}+\Omega_{1}\right) & 0 \\
0 & -\omega_{1}\left(3 \omega_{2}+\Omega_{2}\right) & \omega_{1}\left(3 \omega_{3}+\Omega_{3}\right) \\
\omega_{2}\left(3 \omega_{1}+\Omega_{1}\right) & 0 & -\omega_{2}\left(3 \omega_{3}+\Omega_{3}\right) \\
-\omega_{3}\left(3 \omega_{1}+\Omega_{1}\right) & \omega_{3}\left(3 \omega_{2}+\Omega_{2}\right) & 0
\end{array}\right]^{T}
$$

For any $\boldsymbol{\xi}=\left(\xi_{1}, \xi_{2}, \xi_{3}\right) \in \mathbb{R}^{3}$, we define

$$
\tilde{\Psi}(\omega, \Omega, \xi)=\Psi(\omega, \Omega)-\Psi(\xi, \Omega)
$$

After some tedious yet straightforward algebra, we can find another convenient expression for $\tilde{\Psi}$ 
given by

$$
\tilde{\Psi}(\omega, \Omega, \xi)=H(\omega, \Omega, \xi)\left(I_{3} \otimes(\omega-\boldsymbol{\xi})\right),
$$

where the notation $\otimes$ represents the Kronecker product and $H(\omega, \Omega, \xi)$ is defined as

$$
H(\omega, \Omega, \boldsymbol{\xi})=\left[\begin{array}{lll}
H_{11} & H_{12} & H_{13} \\
H_{21} & H_{22} & H_{23}
\end{array}\right]
$$

with the block matrices

$$
\begin{array}{rlrl}
H_{11} & =\left[\begin{array}{ccc}
0 & 0 & 0 \\
0 & -\left(\omega_{3}+\Omega_{3}\right) & -\xi_{2} \\
0 & \xi_{3} & \left(\omega_{2}+\Omega_{2}\right)
\end{array}\right], & H_{21}=\left[\begin{array}{ccc}
0 & 0 & 0 \\
3 \xi_{2} & \left(3 \omega_{1}+\Omega_{1}\right) & 0 \\
-3 \xi_{3} & 0 & -\left(3 \omega_{1}+\Omega_{1}\right)
\end{array}\right], \\
H_{12} & =\left[\begin{array}{ccc}
\left(\omega_{3}+\Omega_{3}\right) & 0 & \xi_{1} \\
0 & 0 & 0 \\
-\xi_{3} & 0 & -\left(\omega_{1}+\Omega_{1}\right)
\end{array}\right], & H_{22} & =\left[\begin{array}{ccc}
-\left(3 \omega_{2}+\Omega_{2}\right) & -3 \xi_{1} & 0 \\
0 & 0 & 0 \\
0 & 3 \xi_{3} & \left(3 \omega_{2}+\Omega_{2}\right)
\end{array}\right], \\
H_{13} & =\left[\begin{array}{ccc}
-\left(\omega_{2}+\Omega_{2}\right) & -\xi_{1} & 0 \\
\xi_{2} & \left(\omega_{1}+\Omega_{1}\right) & 0 \\
0 & 0 & 0
\end{array}\right], & H_{23}=\left[\begin{array}{ccc}
\left(3 \omega_{3}+\Omega_{3}\right) & 3 \xi_{1} \\
0 & -\left(3 \omega_{3}+\Omega_{3}\right) & -3 \xi_{2} \\
0 & 0 & 0
\end{array}\right] .
\end{array}
$$

For notational simplicity, we additionally define

$$
\boldsymbol{\mu}\left(k_{v}, \mathbf{y}, \omega, \Omega\right)=\boldsymbol{\mu}_{1}\left(k_{v}, \mathbf{y}, \omega\right)+\boldsymbol{\mu}_{2}(\omega, \Omega)
$$

and

$$
\tilde{\boldsymbol{\mu}}\left(k_{v}, \mathbf{y}, \omega, \Omega, \boldsymbol{\xi}\right)=\boldsymbol{\mu}\left(k_{v}, \mathbf{y}, \omega, \Omega\right)-\boldsymbol{\mu}\left(k_{v}, \mathbf{y}, \boldsymbol{\xi}, \Omega\right)
$$

Note that $\tilde{\boldsymbol{\mu}}$ becomes zero when $\boldsymbol{\xi}$ converges to $\omega$ by definition. 


\section{A. Filter-Free Method}

Let us propose

$$
\boldsymbol{\beta}=\Gamma\left(\tilde{\mu}\left(k_{v}, \mathbf{y}, \omega, \Omega, \Omega\right)-\Psi^{T}(\Omega, \Omega) \delta \omega\right)
$$

where $\Gamma \in \mathbb{R}^{6 \times 6}$ is a positive definite matrix. The corresponding parameter update law is then given by

$$
\dot{\hat{\boldsymbol{\theta}}}=-\Gamma\left[\boldsymbol{\mu}^{\prime}+\Psi^{T}(\Omega, \Omega) \dot{\Omega}-\dot{\Psi}^{T}(\Omega, \Omega) \delta \boldsymbol{\omega}+\left(\Phi^{T}(\mathbf{x}, \omega, \Omega)+\tilde{\Psi}^{T}(\omega, \Omega, \Omega)\right)\left(-k_{p} \delta \mathbf{q}_{v}-k_{v} \delta \boldsymbol{\omega}+\dot{\Omega}\right)\right]
$$

where

$$
\boldsymbol{\mu}^{\prime}=\dot{\tilde{\mu}}-\frac{\partial \boldsymbol{\mu}\left(k_{v}, \mathbf{y}, \omega, \Omega\right)}{\partial \omega} \dot{\omega}
$$

so that the time derivative of the parameter estimate error becomes

$$
\begin{aligned}
\dot{\tilde{\boldsymbol{\theta}}} & =\dot{\hat{\boldsymbol{\theta}}}+\dot{\boldsymbol{\beta}} \\
& =-\Gamma(\Phi+\tilde{\Psi})^{T} J^{-1} \Phi \tilde{\boldsymbol{\theta}}
\end{aligned}
$$

Remark 1. A systematic way of constructing $\boldsymbol{\beta}$ is suggested in [12]. This work, however, suggests another way by defining the regressor matrix as a sum of solvable and non-solvable parts such that only the non-solvable part creates the error $\tilde{\Psi}$. It is beneficial because the structure of $\Psi$ is simplified so that it does not depend on other variables like $k_{v}$ and $\mathbf{q}$ for this particular application.

To deal with the perturbation $\tilde{\Psi}$, we introduce the scaled estimate error defined as

$$
\mathbf{z}(t)=\frac{1}{r(t)} \tilde{\boldsymbol{\theta}}(t)
$$

The scaling factor $r \in \mathbb{R}$ is the state of the differential equation

$$
\dot{r}(t)=k_{r} \Gamma_{M}\|\tilde{\Psi}(\omega(t), \Omega(t), \Omega(t))\|_{2}^{2} r(t), r(0)=1,
$$


where $k_{r}>1 /\left(4 J_{m}\right)$ is the gain and $\Gamma_{M}$ is the largest eigenvalue of $\Gamma$. Since $r(t)$ is monotonically increasing and strictly positive for all $t \geq 0$, Eq. (42) is a valid definition. Note that Eq. (43) is implementable as $J_{m}$ is assumed to be known. By differentiating Eq. (42) with respect to time along the trajectories of Eqs. (41) and (43), we obtain

$$
\dot{\mathbf{z}}=-\Gamma\left(\Phi^{T}+\tilde{\Psi}^{T}\right) J^{-1} \Phi \mathbf{z}-k_{r} \Gamma_{M}\|\tilde{\Psi}\|_{2}^{2} \mathbf{z}
$$

Now, consider a Lyapunov-like function

$$
V_{1}(t)=\frac{1}{2} \mathbf{z}^{T}(t) \Gamma^{-1} \mathbf{z}(t)
$$

Utilizing the inequalities

$$
\|\tilde{\Psi}\|_{2}^{2} \geq J_{m}\left\|J^{-1 / 2} \tilde{\Psi}\right\|_{2}^{2}
$$

and

$$
\mathbf{z}^{T} \Gamma^{-1} \mathbf{z} \geq \frac{1}{\Gamma_{M}}\|\mathbf{z}\|_{2}^{2}
$$

the upper bound for the time derivative of $V_{1}$ is obtained as

$$
\dot{V}_{1} \leq-\left[\begin{array}{c}
\left\|J^{-1 / 2} \Phi \mathbf{z}\right\|_{2} \\
\left\|J^{-1 / 2} \tilde{\Psi}\right\|_{2}\|\mathbf{z}\|_{2}
\end{array}\right]^{T} P\left[\begin{array}{c}
\left\|J^{-1 / 2} \Phi \mathbf{z}\right\|_{2} \\
\left\|J^{-1 / 2} \tilde{\Psi}\right\|_{2}\|\mathbf{z}\|_{2}
\end{array}\right]
$$

where

$$
P=\left[\begin{array}{cc}
1 & -\frac{1}{2} \\
-\frac{1}{2} & k_{r} J_{m}
\end{array}\right]
$$

is a positive definite matrix. Observe that the equilibrium $\mathbf{z}=\mathbf{0}$ of Eq. (44) is uniformly globally stable and accordingly $\mathbf{z}(t)$ is uniformly bounded for all $t \geq 0$.

Next, consider the Lyapunov-like function

$$
V_{2}(t)=k_{p}\left[\delta \mathbf{q}_{v}^{T}(t) \delta \mathbf{q}_{v}(t)+\left(1-\delta q_{0}(t)\right)^{2}\right]+\frac{1}{2} \delta \omega^{T}(t) \delta \omega(t)+\zeta V_{1}(t)
$$


where $\zeta$ is a positive constant to be determined. Taking the time derivative with respect to time yields

$$
\dot{V}_{2}(t)=-k_{v}(t) \delta \omega^{T}(t) \delta \omega(t)-r(t) \delta \omega^{T}(t) J^{-1} \Phi(t) z(t)+\zeta \dot{V}_{1}(t)
$$

For the second cross term in the right-hand side of $\dot{V}_{2}$ to be dominated by the first term, we propose the dynamic gain of the form

$$
k_{v}(t)=k \rho(t) r^{2}(t)
$$

where $k$ is a positive scalar and $\rho(t)>0$ is a monotonically decreasing function that is lower bounded by the positive constant $\rho^{*}$ for all $t \geq 0$ such that $\lim _{t \rightarrow \infty} \rho(t)=\rho^{*}$. Once the proposed dynamic gain is plugged in, the upper bound for $\dot{V}_{2}$ is obtained as

$$
\dot{V}_{2} \leq-\frac{k \rho^{*} r^{2}}{2}\|\delta \omega\|_{2}^{2}-\left[\begin{array}{c}
\left\|r J^{-1 / 2} \delta \omega\right\|_{2} \\
\left\|J^{-1 / 2} \Phi \mathbf{z}\right\|_{2} \\
\left\|J^{-1 / 2} \tilde{\Psi}\right\|_{2}\|\mathbf{z}\|_{2}
\end{array}\right]^{T} Q\left[\begin{array}{c}
\left\|r J^{-1 / 2} \delta \omega\right\|_{2} \\
\left\|J^{-1 / 2} \Phi \mathbf{z}\right\|_{2} \\
\left\|J^{-1 / 2} \tilde{\Psi}\right\|_{2}\|\mathbf{z}\|_{2}
\end{array}\right]
$$

where

$$
Q=\left[\begin{array}{ccc}
\frac{k \rho^{*} J_{m}}{2} & -\frac{1}{2} & 0 \\
-\frac{1}{2} & \zeta & -\frac{\zeta}{2} \\
0 & -\frac{\zeta}{2} & \zeta k_{r} J_{m}
\end{array}\right]
$$

If we choose $\zeta>2 k_{r} J_{m} /\left[k \rho^{*}\left(4 k_{r} J_{m}-1\right)\right], Q$ becomes positive definite, and thus, $V_{2}$ is said to be non-increasing. At this point, however, we cannot conclude anything meaningful because the boundedness of $r(t)$ is not established yet. In order to establish $r \in L_{\infty}$, let us recall Eq. (43) and consider the solution $r(t)$ given by

$$
r(t)=\exp \left(k_{r} \Gamma_{M} \int_{0}^{t}\|\Psi(\omega(\sigma), \Omega(\sigma))-\Psi(\Omega(\sigma), \Omega(\sigma))\|_{2}^{2} d \sigma\right) .
$$

$* * * * * * * * * * * * *$ 
Suppose $r(t)$ has a finite escape time at $t=t^{*}>0$. Then

$$
\sup _{t \in\left[0, t^{*}\right)} \int_{0}^{t}\|\tilde{\Psi}(\sigma)\|_{2}^{2} d \sigma=\infty
$$

Since $r(t) \geq r(0)=1, \dot{V}_{2}$ is upper bounded by

$$
\dot{V}_{2}(t) \leq-\frac{k \rho^{*}}{2}\|\delta \omega(t)\|_{2}^{2}
$$

for $t \in\left[0, t^{*}\right)$. Integrating both sides yields

$$
\int_{0}^{t}\|\delta \omega(\sigma)\|_{2}^{2} d \sigma \leq \frac{2}{k \rho^{*}}\left(V_{2}(0)-V_{2}(t)\right) .
$$

Since $V_{2}$ is nonnegative,

$$
\int_{0}^{t}\|\delta \omega(\sigma)\|_{2}^{2} d \sigma \leq \frac{2}{k \rho^{*}} V_{2}(0) .
$$

Also, since $\delta \omega(t)$ is concluded to be bounded for $t \in\left[0, t^{*}\right]$ and $\Omega(t)$ is bounded by the assumption on the reference signals, there is a positive number $L_{\Psi}$ such that

$$
\|H(\omega, \Omega, \Omega)\|_{2} \leq L_{\Psi}
$$

for $t \in\left[0, t^{*}\right]$. However, the inequalities

$$
\begin{aligned}
\int_{0}^{t}\|\tilde{\Psi}(\sigma)\|_{2}^{2} d \sigma & \leq \int_{0}^{t}\|H(\sigma)\|_{2}^{2}\|\delta \omega(\sigma)\|_{2}^{2} d \sigma \\
& \leq \frac{2 L_{\Psi}^{2} V_{2}(0)}{k \rho^{*}}
\end{aligned}
$$

hold for all $t \in\left[0, t^{*}\right)$. This contradict the hypothesis (56).

$* * * * * * * * * * * * * * * * * * * * * * *$

Thus, $r(t)$ has neither a finite escape time nor diverges to infinity in the limit. Now Eq. (53) is 
valid for all $t \geq 0$, and there exist positive constants $c_{1}$ and $c_{2}$ such that

$$
\dot{V}_{2} \leq-c_{1}\left\|r J^{-1 / 2} \delta \omega\right\|_{2}^{2}-c_{2}\left\|J^{-1 / 2} \Phi \mathbf{z}\right\|_{2}^{2}
$$

Since $r \in L_{\infty}$, it is concluded that $\tilde{\boldsymbol{\theta}} \in L_{\infty}, \delta \boldsymbol{\omega} \in L_{2} \cap L_{\infty}$ and $\delta \dot{\boldsymbol{\omega}} \in L_{\infty}$. Then invoking Barbalat's lemma allows us to conclude

$$
\lim _{t \rightarrow \infty} \delta \omega(t)=\mathbf{0}
$$

Lastly, let us show the convergence of $\delta \mathbf{q}_{v}$ to zero by partially stritifying the function $V_{2}(t)$. [26] Consider the strictified function

$$
V_{3}(t)=c V_{2}(t)+\delta \mathbf{q}_{v}^{T}(t) \delta \omega(t)
$$

where

$$
c>\max \left\{\sqrt{\frac{1}{2 k_{p}}}, \sup _{t \geq 0} \frac{\left(k_{p}+k_{v}^{2}\right)\|J\|_{2}}{2 k_{p} r^{2} c_{1}}, \sup _{t \geq 0} \frac{r^{2}}{2 k_{p} J_{m} c_{2}}\right\} .
$$

Since $c>\sqrt{\frac{1}{2 k_{p}}}, V_{3}$ is lower bonded by the following non-negative function:

$$
V_{3} \geq\left[\begin{array}{c}
\delta \mathbf{q}_{v} \\
\delta \omega
\end{array}\right]^{T}\left(\left[\begin{array}{cc}
c k_{p} & \frac{1}{2} \\
\frac{1}{2} & \frac{c}{2}
\end{array}\right] \otimes I_{3}\right)\left[\begin{array}{c}
\delta \mathbf{q}_{v} \\
\delta \omega
\end{array}\right]+c \zeta V_{1}
$$

Using the inequalities

$$
-k_{v} \delta \mathbf{q}_{v}^{T} \delta \omega \leq \frac{k_{p}}{2}\left\|\delta \mathbf{q}_{v}\right\|_{2}^{2}+\frac{k_{v}^{2}}{2 k_{p}}\|\delta \omega\|_{2}^{2}
$$

and

$$
\left\|S\left(\mathbf{q}_{v}\right)+q_{0} I_{3}\right\|_{2} \leq\|E(\mathbf{q})\|_{2},
$$

the upper bound of $\dot{V}_{3}$ is obtained as

$$
\dot{V}_{3} \leq c \dot{V}_{2}-\frac{k_{p}}{2}\left\|\delta \mathbf{q}_{v}\right\|_{2}^{2}+\left(\frac{k_{p}+k_{v}^{2}}{2 k_{p}}\right)\|\delta \omega\|_{2}^{2}+\left\|r J^{-1 / 2} \delta \mathbf{q}_{v}\right\|_{2}\left\|J^{-1 / 2} \Phi \mathbf{z}\right\|_{2}
$$


Furthermore, aided by the inequalities $\frac{1}{\|J\|_{2}}\|\mathbf{v}\|_{2}^{2} \leq \mathbf{v}^{T} J^{-1} \mathbf{v} \leq \frac{1}{J_{m}}\|\mathbf{v}\|_{2}^{2}$ for any $\mathbf{v} \in \mathbb{R}^{3}$, we finally have

$$
\dot{V}_{3} \leq-c_{1}\left(c-\frac{\left(k_{p}+k_{v}^{2}\right)\|J\|_{2}}{2 k_{p} r^{2} c_{1}}\right)\left\|r J^{-1 / 2} \delta \omega\right\|_{2}^{2}-\left[\begin{array}{c}
\left\|r J^{-1 / 2} \delta \mathbf{q}_{v}\right\|_{2} \\
\left\|J^{-1 / 2} \Phi \mathbf{z}\right\|_{2}
\end{array}\right]^{T} R\left[\begin{array}{c}
\left\|r J^{-1 / 2} \delta \mathbf{q}_{v}\right\|_{2} \\
\left\|J^{-1 / 2} \Phi \mathbf{z}\right\|_{2}
\end{array}\right],
$$

where

$$
R=\left[\begin{array}{cc}
\frac{k_{p} J_{m}}{2 r^{2}} & -\frac{1}{2} \\
-\frac{1}{2} & c c_{2}
\end{array}\right]
$$

is a positive definite matrix. Again, since $r(t)$ is bounded and $J$ is positive definite, we can conclude $\delta \mathbf{q}_{v} \in L_{2} \cap L_{\infty}$ and $\delta \dot{\mathbf{q}}_{v} \in L_{\infty}$. Therefore, by Barbalat's lemma,

$$
\lim _{t \rightarrow \infty} \delta \mathbf{q}_{v}(t)=\mathbf{0}
$$

To complete our design, let us propose the following scalar differential equations for $\rho(t)$ :

$$
\begin{aligned}
& \dot{\rho}_{1}(t)=-2 k_{r} \Gamma_{M}\left(k_{1}+(v+1)\|\tilde{\Psi}(t)\|_{2}^{2}\right)\left(\rho_{1}(t)-\frac{1}{r^{2}(t)}-\epsilon \rho_{2}(t)\right), \\
& \dot{\rho}_{2}(t)=-k_{2} \rho_{3}^{2}(t) \rho_{2}(t) \\
& \dot{\rho}_{3}(t)=-k_{3} \rho_{3}^{2}(t)
\end{aligned}
$$

with the initial conditions $\rho_{1}(0)=1+\epsilon^{2}, \rho_{2}(0)=\epsilon$ and $\rho_{3}(0)>0$, where $\epsilon$ is a sufficiently small positive constant, and $v, k_{1}, k_{2}, k_{3}>0$. Note that $\rho_{2}(t)$ is monotonically decreasing and lower bounded by a positive constant because $\rho_{3} \in L_{2}$, and thus, $\lim _{t \rightarrow \infty} \rho_{2}(t)>0$. Moreover, since $\rho_{1}(t)$ is also monotonically decreasing and lower bounded by the positive constant $\lim _{t \rightarrow \infty} \epsilon \rho_{2}(t)$, the choice $\rho(t)=\rho_{1}(t)$ will result in $\lim _{t \rightarrow \infty}\left(\delta \mathbf{q}_{v}(t), \delta \boldsymbol{\omega}(t)\right)=(\mathbf{0}, \mathbf{0})$. Alternatively, one can propose $\rho(t)=1$ for all $t \geq 0$ since a constant function satisfies the condition on $\rho(t)$ so that only one dimensional dynamic extension is required, but the gain $k_{v}(t)$ increases monotonically.

We can also remove the monotonically increasing scaling factor $r(t)$ from the closed-loop dy- 
namics by introducing the following transformations:

$$
\begin{aligned}
& \eta_{1}(t)=\rho_{1}(t) r^{2}(t), \\
& \eta_{2}(t)=\rho_{2}(t) r^{2}(t), \\
& \eta_{3}(t)=\rho_{3}(t)
\end{aligned}
$$

with the initial conditions $\eta_{1}(0)=1+\epsilon^{2}, \eta_{2}(0)=\epsilon$ and $\eta_{3}(0)>0$. Then the extended dynamics become

$$
\begin{aligned}
& \dot{\eta}_{1}(t)=-2 k_{r} \Gamma_{M}\left[\left(k_{1}+v\|\tilde{\Psi}(t)\|_{2}^{2}\right) \eta_{1}(t)-\left(k_{1}+(v+1)\|\tilde{\Psi}(t)\|_{2}^{2}\right)\left(1+\epsilon \eta_{2}(t)\right)\right] \\
& \dot{\eta}_{2}(t)=-2 k_{r} \Gamma_{M}\left(k_{2} \eta_{3}^{2}(t)-\|\tilde{\Psi}(t)\|_{2}^{2}\right) \eta_{2}(t) \\
& \dot{\eta}_{3}(t)=-k_{3} \eta_{3}^{2}(t)
\end{aligned}
$$

and consequently, the dynamic gain is expressed as

$$
k_{v}(t)=k \eta_{1}(t) .
$$

Choosing proper values for $k_{2}$ and $k_{3}$ such that the first term in the right hand side of Eq. (80) dominates the other term enables $\eta_{2}(t)$ to converge to a sufficiently small positive number. Then $\eta_{1}(t)$ will converge close to the unity. In other words, we can select $k_{2}$ and $1 / k_{3}$ to be arbitrarily large to render $\eta_{2}(t)$ arbitrarily small and still ensure the boundedness of $r(t)$. Now $k_{2}$ and $k_{3}$ are another parameters we can tune to control the convergence of $k_{v}(t)$. Observe that since

$$
1 \leq \eta_{1}(t) \leq \frac{\left(k_{1}+(v+1)\|\tilde{\Psi}(t)\|_{2}^{2}\right)\left(1+\epsilon \eta_{2}(t)\right)}{k_{1}+v\|\tilde{\Psi}(t)\|_{2}^{2}} \leq \frac{v+1}{v}\left(1+\epsilon \eta_{2}(t)\right)
$$

for all $t \geq 0$, the upper bound of the dynamic gain $k_{v}(t)$ can be adjusted by the parameter $v$ as long as $\eta_{2}$ converges to a small number.

Remark 2. Practically, the initial condition for $\eta_{3}(t)$ can be reset such that $k_{2} \eta_{3}^{2}(t)>\|\tilde{\Psi}(t)\|_{2}^{2}$ at $t=t^{*}$ for any $t^{*} \geq 0$ whenever needed so that $\eta_{2}(t)$ decreases. Since the infinite tail of the signal 
$\eta_{3}(t)$ still satisfies the $L_{2}$ condition, we can reset the dynamics finitely many times.

Remark 3. To ensure the boundedness of $r(t), k_{v}(t)$ must be proportional to $r^{2}(t)$. Ideally, only one dimensional dynamic extension for $r(t)$ achieves the I\&I adaptive tracking control. However, the dynamic gain includes $r^{2}(t)$, and thus, it is possible for the gain to grow to an arbitrarily large number with a constant $\rho$. This is undesirable for both the implementation of the scaling factor dynamics and the robustness properties of the closed-loop system. To prevent the high gain feedback control, three more dynamics (73), (74) and (75) are introduced so that monotonically increasing $r(t)$ is removed from the closed-loop states giving us more flexibility to tune the range of the dynamic gain. Overall, only 3 more dynamics are required to overcome the integrability obstacle, which is a significant order-reduction compared to the existing I\&I controller with an 18-dimensional regressor filter and a 3-dimensional state filter. [9]

\section{B. Filter Method}

The gain $k_{v}(t)$ described in the filter-free method can be set to a constant if a filter for $\omega(t)$ is introduced. Let $\hat{\boldsymbol{\omega}}(t)$ be the filter state whose dynamics are to be determined such that $\lim _{t \rightarrow \infty} \tilde{\boldsymbol{\omega}}(t)=$ $\mathbf{0}$ and $\tilde{\boldsymbol{\theta}} \in L_{\infty}$, where $\tilde{\boldsymbol{\omega}}(t)=\boldsymbol{\omega}(t)-\hat{\boldsymbol{\omega}}(t)$. Previously, the perturbation term $\tilde{\Psi}(t)$ was created using the reference rate $\Omega(t)$. Once a filter is considered, $\hat{\omega}(t)$ can replace $\Omega(t)$ so that we have

$$
\begin{aligned}
\tilde{\Psi}(t) & =\tilde{\Psi}(\omega(t), \Omega(t), \hat{\omega}(t)) \\
& =\Psi(\omega(t), \Omega(t))-\Psi(\hat{\omega}(t), \Omega(t)) .
\end{aligned}
$$

The estimator design process is similar to the filter-free method. We first propose

$$
\boldsymbol{\beta}=\Gamma\left(\tilde{\boldsymbol{\mu}}\left(k_{v}, \mathbf{y}, \boldsymbol{\omega}, \Omega, \hat{\boldsymbol{\omega}}\right)-\Psi^{T}(\hat{\boldsymbol{\omega}}, \Omega) \tilde{\boldsymbol{\omega}}\right)
$$


and

$$
\dot{\hat{\boldsymbol{\theta}}}=-\Gamma\left[\boldsymbol{\mu}^{\prime}+\Psi^{T}(\hat{\boldsymbol{\omega}}, \Omega) \dot{\hat{\boldsymbol{\omega}}}-\dot{\Psi}^{T}(\hat{\boldsymbol{\omega}}, \Omega) \tilde{\boldsymbol{\omega}}+\left(\Phi^{T}(\mathbf{x}, \boldsymbol{\omega}, \Omega)+\tilde{\Psi}^{T}(\boldsymbol{\omega}, \Omega, \hat{\boldsymbol{\omega}})\right)\left(-k_{p} \delta \mathbf{q}_{v}-k_{v} \delta \boldsymbol{\omega}+\dot{\Omega}\right)\right]
$$

where

$$
\boldsymbol{\mu}^{\prime}=\dot{\tilde{\mu}}-\frac{\partial \boldsymbol{\mu}\left(k_{v}, \mathbf{y}, \omega, \Omega\right)}{\partial \omega} \dot{\omega}
$$

so that the parameter error dynamics become

$$
\dot{\tilde{\boldsymbol{\theta}}}=-\Gamma(\Phi+\tilde{\Psi})^{T} J^{-1} \Phi \tilde{\boldsymbol{\theta}}
$$

The dynamic equation for the scaling factor $r(t)$ is then changed to

$$
\dot{r}(t)=k_{r} \Gamma_{M}\|\tilde{\Psi}(\omega(t), \Omega(t), \hat{\omega}(t))\|_{2}^{2} r(t), r(0)=1 .
$$

to obtain

$$
\dot{\mathbf{z}}=-\Gamma\left(\Phi^{T}+\tilde{\Psi}^{T}\right) J^{-1} \Phi \mathbf{z}-k_{r} \Gamma_{M}\|\tilde{\Psi}\|_{2}^{2} \mathbf{z}
$$

Next, propose the filter dynamics

$$
\dot{\hat{\omega}}(t)=k_{f}(t) \tilde{\omega}(t)-k_{p} \delta \mathbf{q}_{v}(t)-k_{v} \delta \omega(t)+\dot{\Omega}(t),
$$

where the filter gain $k_{f}(t)>0$ contains the scaling factor. Since the dynamic gain does not appear in the control law, the local Lipschitz condition for $\Psi$ cannot be used to show the boundedness of $r(t)$. So we choose

$$
k_{f}(t)=\left(k+k_{H}\|H(\omega, \Omega, \hat{\omega})\|_{2}^{2}\right) \rho(t) r^{2}(t)
$$

and consider the Lyapunov-like function

$$
W_{1}(t)=\frac{1}{2} \tilde{\boldsymbol{\omega}}^{T}(t) \tilde{\boldsymbol{\omega}}(t)+\frac{\zeta}{2} z^{T}(t) \Gamma^{-1} z(t)+\frac{k_{H} \rho^{*}}{k_{r} \Gamma_{M}} r^{2}(t)
$$


where the gain $k$ and $k_{H}$ are positive constants [12]. When we differentiate $W_{1}(t)$ with respect to time, the upper bound for $\dot{W}_{1}(t)$ is obtained as

$$
\dot{W}_{1} \leq-\frac{k \rho^{*} r^{2}}{2}\|\tilde{\boldsymbol{\omega}}\|_{2}^{2}-\left[\begin{array}{c}
\left\|r J^{-1 / 2} \tilde{\boldsymbol{\omega}}\right\|_{2} \\
\left\|J^{-1 / 2} \Phi \mathbf{z}\right\|_{2} \\
\left\|J^{-1 / 2} \tilde{\Psi}\right\|_{2}\|\mathbf{z}\|_{2}
\end{array}\right]^{T} Q\left[\begin{array}{c}
\left\|r J^{-1 / 2} \tilde{\boldsymbol{\omega}}\right\|_{2} \\
\left\|J^{-1 / 2} \Phi \mathbf{z}\right\|_{2} \\
\left\|J^{-1 / 2} \tilde{\Psi}\right\|_{2}\|\mathbf{z}\|_{2}
\end{array}\right]
$$

The dynamic gain with an extra term directly ensures $r \in L_{\infty}$ in this case. Consequently, by Barbalat's lemma, the convergence of the filter error to zero is guaranteed, i.e.,

$$
\lim _{t \rightarrow \infty} \tilde{\omega}(t)=\mathbf{0}
$$

The convergence of the tracking errors to zero is proved by considering the function

$$
W_{2}(t)=k_{p} a_{1}\left[\delta \mathbf{q}_{v}^{T}(t) \delta \mathbf{q}_{v}(t)+\left(1-\delta q_{0}(t)\right)^{2}\right]+\frac{a_{1}}{2} \delta \omega^{T}(t) \delta \boldsymbol{\omega}(t)+a_{2} W_{1}(t)+\delta \mathbf{q}_{v}^{T}(t) \delta \omega(t)
$$

for sufficiently large $a_{1}, a_{2}>0$. Here we omit the proof since the procedure is almost the same as that in the filter-free case. Also, $r$-dynamics can be removed through the dynamic extensions (79), (80) and (81). Then the gain is given by

$$
k_{f}(t)=\left(k+k_{H}\|H(\omega, \Omega, \hat{\omega})\|_{2}^{2}\right) \eta_{1}(t) .
$$

Remark 4. The boundedness of $r(t)$ is established by adding an extra term in the dynamic gain. If we apply this approach to the filter-free case, the dynamic gain would be of the form

$$
k_{v}(t)=\left(k+k_{H}\|H(\omega, \Omega, \Omega)\|_{2}^{2}\right) \eta_{1}(t)
$$

However, this is not applicable since $\mu^{\prime}(t)$ defined in Eq. (40) includes $\dot{k}_{v}(t)$, and therefore, requires the unavailable state $\dot{\omega}(t)$ for the estimator implementation. 
Remark 5. The advantage of the filter method is that we can always choose $\hat{\omega}(0)=\omega(0)$. So the transient response of the closed-loop system would be better than filter-free method when the initial tracking error is large. Moreover, since the high gain term only appears in the filter dynamics, the required control effort can be less than that of the filter-free controller.

\section{Application to Robot Manipulator Control}

The same design process can be applied to Euler-Lagrange equations, which describe the evolution of a mechanical system subject to holonomic constraints [27]. In fact, the dynamic model for the rigid-body rotational motion with the quaternion kinematics can be converted to a similar form of the Euler-Lagrange equations by differentiating the following equation with respect to time [18]:

$$
\delta \dot{\mathbf{q}}_{v}(t)=\frac{1}{2} F(\delta \mathbf{q}(t)) \delta \omega(t) .
$$

In this section, motivated by this analogy, we further develop and generalize the theory for $n$ link robot manipulator systems. Instead of following the entire design procedure suggested in the previous section, we only introduce necessary conditions to apply the dynamic scaling based I\&I methodology.

\section{A. Dynamic Equations}

Let us consider the equations of motion for an $n$-link robot given by

$$
\begin{aligned}
\dot{\mathbf{x}}_{1}(t) & =\mathbf{x}_{2}(t) \\
M\left(\mathbf{x}_{1}(t)\right) \dot{\mathbf{x}}_{2}(t) & =\mathbf{u}(t)-C\left(\mathbf{x}_{1}(t), \mathbf{x}_{2}(t)\right) \mathbf{x}_{2}(t)-\mathbf{g}\left(\mathbf{x}_{1}(t)\right),
\end{aligned}
$$

where $\mathbf{x}_{1}(t), \mathbf{x}_{2}(t) \in \mathbb{R}^{n}$ are the joint angle and rate, and $\mathbf{u}(t) \in \mathbb{R}^{n}$ is the control torque at each joint. $M\left(\mathbf{x}_{1}(t)\right)$ is the inertia matrix, $C\left(\mathbf{x}_{1}(t), \mathbf{x}_{2}(t)\right)$ is the centripetal-Coriolis matrix and $\mathbf{g}\left(\mathbf{x}_{1}(t)\right)$ is the gravity vector satisfying the following three important structural properties [27]:

Property 1 (Skew-symmetry property). The matrix $\dot{M}\left(\mathbf{x}_{1}\right)-2 C\left(\mathbf{x}_{1}, \mathbf{x}_{2}\right)$ is skew-symmetric, that is, 
the equality

$$
\boldsymbol{\xi}^{T}\left(\dot{M}\left(\mathbf{x}_{1}\right)-2 C\left(\mathbf{x}_{1}, \mathbf{x}_{2}\right)\right) \boldsymbol{\xi}=0
$$

holds for all $\boldsymbol{\xi} \in \mathbb{R}^{n}$.

Property 2 (Bounds on the inertia matrix). The inertia matrix $M\left(\mathbf{x}_{1}\right)$ is positive definite and its eigenvalues are bounded for any fixed value of $\mathbf{x}_{1}$. Let $0<\lambda_{1}\left(\mathbf{x}_{1}\right) \leq \ldots \leq \lambda_{n}\left(\mathbf{x}_{1}\right)$ denote the $n$ eigenvalues of $M\left(\mathbf{x}_{1}\right)$. Then the inertia matrix satisfies

$$
\lambda_{1}\left(\mathbf{x}_{1}\right) I_{n} \leq M\left(\mathbf{x}_{1}\right) \leq \lambda_{n}\left(\mathbf{x}_{1}\right) I_{n}
$$

where $I_{n}$ is the $n$-dimensional identity matrix and the matrix inequality $B \leq A$ means $A-B$ is positive semidefinite. If $\mathbf{x}_{1} \in L_{\infty}$ or all joints are revolute, $M\left(\mathbf{x}_{1}\right)$ is uniformly bounded by positive constants $M_{m}$ and $M_{M}$ such that

$$
M_{m} I_{n} \leq M\left(\mathbf{x}_{1}\right) \leq M_{M} I_{n}
$$

Property 3 (Linearity in the parameters). The dynamic equations are linear in the inertial parameters such that there exists an $n \times p$ regressor function $Y\left(\mathbf{x}_{1}, \mathbf{x}_{2}, \dot{\mathbf{x}}_{2}\right)$, which is completely known and satisfies

$$
M\left(\mathbf{x}_{1}\right) \dot{\mathbf{x}}_{2}+C\left(\mathbf{x}_{1}, \mathbf{x}_{2}\right) \mathbf{x}_{2}+\mathbf{g}\left(\mathbf{x}_{1}\right)=Y\left(\mathbf{x}_{1}, \mathbf{x}_{2}, \dot{\mathbf{x}}_{2}\right) \boldsymbol{\theta}^{*}
$$

where $\boldsymbol{\theta}^{*} \in \mathbb{R}^{p}$ is the parameter vector.

The objective is to design a tracking adaptive controller when the system parameters $\boldsymbol{\theta}^{*}$ are uncertain. Let $\mathbf{x}_{r}(t)=\left(\mathbf{x}_{r 1}(t), \mathbf{x}_{r 2}(t)\right) \in \mathbb{R}^{2 n}$ be the reference state to be tracked with the matching condition

$$
\begin{aligned}
\dot{\mathbf{x}}_{r 1}(t) & =\mathbf{x}_{r 2}(t) \\
\dot{\mathbf{x}}_{r 2}(t) & =\mathbf{f}\left(t, \mathbf{x}_{r}(t)\right),
\end{aligned}
$$

where $\mathbf{f}:[0, \infty) \times \mathbb{R}^{2 n} \rightarrow \mathbb{R}^{n}$ is a predefined smooth function that produces bounded values for 
bounded arguments. It is also assumed that the time derivative of the forcing function $\mathbf{f}\left(t, \mathbf{x}_{r}(t)\right)$ is bounded. If error states are defined as

$$
\begin{aligned}
& \mathbf{e}_{1}(t)=\mathbf{x}_{1}(t)-\mathbf{x}_{r 1}(t) \\
& \mathbf{e}_{2}(t)=\mathbf{x}_{2}(t)-\mathbf{x}_{r 2}(t),
\end{aligned}
$$

then the error dynamics are obtained as

$$
\begin{aligned}
& \dot{\mathbf{e}}_{1}=\mathbf{e}_{2} \\
& \dot{\mathbf{e}}_{2}=M^{-1}\left(\mathbf{x}_{1}\right)\left[\mathbf{u}-C\left(\mathbf{x}_{1}, \mathbf{x}_{2}\right) \mathbf{x}_{2}-\mathbf{g}\left(\mathbf{x}_{1}\right)-M\left(\mathbf{x}_{1}\right) \mathbf{f}\right] .
\end{aligned}
$$

Let the target dynamics for $\mathbf{e}_{2}(t)$ be given by

$$
\dot{\mathbf{e}}_{2}(t)=-k_{p} \mathbf{e}_{1}(t)-k_{v}(t) \mathbf{e}_{2}(t)
$$

where $k_{p}>0$ is the static feedback gain for the position error and $k_{v}(t)>0$ is the static or dynamic feedback gain for the velocity error. Then the error dynamics for the velocity error in Eq. (107) can be rewritten as

$$
\dot{\mathbf{e}}_{2}=-k_{p} \mathbf{e}_{1}-k_{\nu} \mathbf{e}_{2}+M^{-1}\left(\mathbf{x}_{1}\right)\left[\mathbf{u}+M\left(\mathbf{x}_{1}\right)\left(k_{p} \mathbf{e}_{1}+k_{\nu} \mathbf{e}_{2}-\mathbf{f}\right)-C\left(\mathbf{x}_{1}, \mathbf{x}_{2}\right) \mathbf{x}_{2}-\mathbf{g}\left(\mathbf{x}_{1}\right)\right] .
$$

\section{B. Parameterization}

By Property 3, there exist functions $\Phi_{1}\left(\mathbf{y}, \mathbf{x}_{1}\right), \Phi_{2}\left(\mathbf{x}_{1}, \mathbf{x}_{2}\right), \Phi_{3}\left(\mathbf{x}_{1}\right) \in \mathbb{R}^{n \times p}$ such that

$$
\begin{aligned}
\Phi_{1}\left(\mathbf{y}, \mathbf{x}_{1}\right) \boldsymbol{\theta}^{*} & =M\left(\mathbf{x}_{1}\right) \mathbf{y} \\
\Phi_{2}\left(\mathbf{x}_{1}, \mathbf{x}_{2}\right) \boldsymbol{\theta}^{*} & =-C\left(\mathbf{x}_{1}, \mathbf{x}_{2}\right) \mathbf{x}_{2} \\
\Phi_{3}\left(\mathbf{x}_{1}\right) \boldsymbol{\theta}^{*} & =-\mathbf{g}\left(\mathbf{x}_{1}\right),
\end{aligned}
$$


where $\mathbf{y}=k_{p} \mathbf{e}_{1}+k_{\nu} \mathbf{e}_{2}-\mathbf{f}$. Once the regressor matrix $\Phi\left(\mathbf{y}, \mathbf{x}_{1}, \mathbf{x}_{2}\right)$ is defined as

$$
\Phi\left(\mathbf{y}, \mathbf{x}_{1}, \mathbf{x}_{2}\right)=\Phi_{1}\left(\mathbf{y}, \mathbf{x}_{1}\right)+\Phi_{2}\left(\mathbf{x}_{1}, \mathbf{x}_{2}\right)+\Phi_{3}\left(\mathbf{x}_{1}\right)
$$

the form of the dynamic equations we are interested in is given by

$$
\begin{aligned}
& \dot{\mathbf{e}}_{1}=\mathbf{e}_{2} \\
& \dot{\mathbf{e}}_{2}=-k_{p} \mathbf{e}_{1}-k_{v} \mathbf{e}_{2}+M^{-1}\left(\mathbf{x}_{1}\right)\left[\mathbf{u}+\Phi\left(\mathbf{y}, \mathbf{x}_{1}, \mathbf{x}_{2}\right) \boldsymbol{\theta}^{*}\right]
\end{aligned}
$$

\section{Partial Differential Equations for I\&I Design}

Suppose there exists $\boldsymbol{\mu}\left(\mathbf{s}, \mathbf{x}_{1}, \mathbf{x}_{2}\right) \in \mathbb{R}^{p}$ for some variable $\mathbf{s}$ independent of $\mathbf{x}_{2}$, which satisfies the PDE

$$
\frac{\partial \boldsymbol{\mu}}{\partial \mathbf{x}_{2}}=\Phi^{T}\left(\mathbf{y}, \mathbf{x}_{1}, \mathbf{x}_{2}\right)
$$

Then the integrability condition is said to be satisfied, and thus, there is an I\&I adaptive controller [3]. However, the existence of $\boldsymbol{\mu}$ is not always guaranteed unless $n=1$. Let $\boldsymbol{\phi}_{i}$ be the $i$ th column of the matrix $\Phi^{T}\left(\mathbf{y}, \mathbf{x}_{1}, \mathbf{x}_{2}\right)$ and $x_{j}$ be the $j$ th component of the vector $\mathbf{x}_{2} \in \mathbb{R}^{n}$. In order for $\mu\left(\mathbf{s}, \mathbf{x}_{1}, \mathbf{x}_{2}\right)$ to exist satisfying Eq. (115), the condition

$$
\frac{\partial \phi_{i}}{\partial x_{j}}-\frac{\partial \phi_{j}}{\partial x_{i}}=0
$$

where $i, j \in\{1,2, \ldots, n\}$ must hold for each $i$ and $j$.

In our formulation, we show that there exists $\boldsymbol{\mu}_{1}\left(k_{v}, \overline{\mathbf{y}}, \mathbf{x}_{1}, \mathbf{x}_{2}\right) \in \mathbb{R}^{p}$ such that

$$
\frac{\partial \mu_{1}}{\partial \mathbf{x}_{2}}=\Phi_{1}^{T}\left(\mathbf{y}, \mathbf{x}_{1}\right)
$$

where $\overline{\mathbf{y}}=k_{p} \mathbf{e}_{1}-k_{v} \mathbf{x}_{r 2}-\mathbf{f}$. To find a solution to Eq. (117), let us establish the following lemma.

Lemma 1. Consider the parametrization defined in Eq. (110). Then, there exists $\boldsymbol{\mu}^{*}\left(\mathbf{x}_{1}, \mathbf{x}_{2}\right) \in \mathbb{R}^{p}$ such that $\partial \boldsymbol{\mu}^{*} / \partial \mathbf{x}_{2}=\Phi_{1}^{T}\left(\mathbf{x}_{2}, \mathbf{x}_{1}\right)$. 
Proof. By Property 3, the element of the $i$ th row and $j$ th column of $M\left(\mathbf{x}_{1}\right)$ is a inner product of the parameter vector $\boldsymbol{\theta}^{*}$ and some vector function $\mathbf{f}_{i j}\left(\mathbf{x}_{1}\right) \in \mathbb{R}^{p}$, i.e.,

$$
M_{i j}=\sum_{k=1}^{p} \theta_{k} f_{i j k}\left(\mathbf{x}_{1}\right)
$$

with $f_{i j k}\left(\mathbf{x}_{1}\right)=f_{j i k}\left(\mathbf{x}_{1}\right)$, where $\theta_{k}$ and $f_{i j k}\left(\mathbf{x}_{1}\right)$ are the $k$ th elements of $\boldsymbol{\theta}^{*}$ and $\mathbf{f}_{i j}$. Define $\boldsymbol{\xi}=M\left(\mathbf{x}_{1}\right) \mathbf{x}_{2}$. Let $x_{2 i}$ and $\xi_{i}$ be the $i$ th elements of the vector $\mathbf{x}_{2}$ and $\xi$. Then, since

$$
\begin{aligned}
\xi_{i} & =\sum_{j=1}^{n} \sum_{k=1}^{p} \theta_{k} f_{i j k}\left(\mathbf{x}_{1}\right) x_{2 j} \\
& =\sum_{k=1}^{p} \theta_{k} \sum_{j=1}^{n} f_{i j k}\left(\mathbf{x}_{1}\right) x_{2 j},
\end{aligned}
$$

the entry in the $k$ th row and $i$ th column of $\Phi_{1}^{T}\left(\mathbf{x}_{2}, \mathbf{x}_{1}\right)$ denoted as $\left[\Phi_{1}^{T}\right]_{k i}$ is obtained as

$$
\left[\Phi_{1}^{T}\right]_{k i}=\sum_{j=1}^{n} f_{i j k}\left(\mathbf{x}_{1}\right) x_{2 j}
$$

Applying the integrability condition (116) to the $i$ th and $l$ th columns of $\Phi_{1}^{T}\left(\mathbf{x}_{2}, \mathbf{x}_{1}\right), \boldsymbol{\phi}_{1 i}$ and $\boldsymbol{\phi}_{1 j}$ respectively, yields

$$
\frac{\partial \boldsymbol{\phi}_{1 i}}{\partial x_{l}}-\frac{\partial \boldsymbol{\phi}_{1 l}}{\partial x_{i}}=\mathbf{0}
$$

which proves the existence of $\boldsymbol{\mu}^{*}$.

Since $\Phi_{1}\left(\mathbf{y}, \mathbf{x}_{1}\right)$ is linear in the second argument, it is decomposed as

$$
\Phi_{1}\left(\mathbf{y}, \mathbf{x}_{1}\right)=\Phi_{1}\left(\overline{\mathbf{y}}, \mathbf{x}_{1}\right)+k_{v} \Phi_{1}\left(\mathbf{x}_{2}, \mathbf{x}_{1}\right)
$$

Then, by Lemma 1, there is $\boldsymbol{\mu}^{*}\left(\mathbf{x}_{1}, \mathbf{x}_{2}\right)$ such that $\partial \boldsymbol{\mu}^{*} / \partial \mathbf{x}_{2}=\Phi_{1}^{T}\left(\mathbf{x}_{2}, \mathbf{x}_{1}\right)$, and thus,

$$
\boldsymbol{\mu}_{1}\left(k_{v}, \overline{\mathbf{y}}, \mathbf{x}_{1}, \mathbf{x}_{2}\right)=\Phi_{1}^{T}\left(\overline{\mathbf{y}}, \mathbf{x}_{1}\right) \mathbf{x}_{2}+k_{v} \mu^{*}\left(\mathbf{x}_{1}, \mathbf{x}_{2}\right)
$$


is a solution to Eq. (117). Also, we can choose

$$
\mu_{3}\left(\mathbf{x}_{1}, \mathbf{x}_{2}\right)=\Phi_{3}^{T}\left(\mathbf{x}_{1}\right) \mathbf{x}_{2}
$$

to have

$$
\frac{\partial \mu_{3}}{\partial \mathbf{x}_{2}}=\Phi_{3}^{T}\left(\mathbf{x}_{1}\right)
$$

However, the existence of $\boldsymbol{\mu}_{2} \in \mathbb{R}^{p}$ such that $\partial \boldsymbol{\mu}_{2} / \partial \mathbf{x}_{2}=\Phi_{2}^{T}\left(\mathbf{x}_{1}, \mathbf{x}_{2}\right)$ is not guaranteed. So we consider a solvable PDE by introducing the function $\Psi\left(\mathbf{x}_{1}, \mathbf{x}_{2}\right)$ satisfying

$$
\frac{\partial \boldsymbol{\psi}_{j}}{\partial x_{i}}-\frac{\partial \boldsymbol{\psi}_{i}}{\partial x_{j}}=\frac{\partial \boldsymbol{\phi}_{2_{i}}}{\partial x_{j}}-\frac{\partial \boldsymbol{\phi}_{2_{j}}}{\partial x_{i}}
$$

where the subscripts $i$ and $j$ for $\psi$ and $\phi_{2}$ indicate the $i$ th and $j$ th columns of the matrices $\Psi^{T}$ and $\Phi_{2}^{T}$ respectively. Then the PDE is modified as follows:

$$
\frac{\partial \mu_{2}\left(\mathbf{x}_{1}, \mathbf{x}_{2}\right)}{\partial \mathbf{x}_{2}}=\Phi_{2}^{T}\left(\mathbf{x}_{1}, \mathbf{x}_{2}\right)+\Psi^{T}\left(\mathbf{x}_{1}, \mathbf{x}_{2}\right)
$$

with a solution $\boldsymbol{\mu}_{2}$. Such $\Psi\left(\mathbf{x}_{1}, \mathbf{x}_{2}\right)$ satisfying Eq. (127) always exists because, for example, we can always select

$$
\Psi\left(\mathbf{x}_{1}, \mathbf{x}_{2}\right)=-\Phi_{2}\left(\mathbf{x}_{1}, \mathbf{x}_{2}\right)
$$

or alternately as

$$
\Psi\left(\mathbf{x}_{1}, \mathbf{x}_{2}\right)=\left[\frac{\partial}{\partial \mathbf{x}_{2}}\left(\Phi_{2}^{T}\left(\mathbf{x}_{1}, \mathbf{x}_{2}\right) \mathbf{x}_{2}\right)\right]^{T}-\Phi_{2}\left(\mathbf{x}_{1}, \mathbf{x}_{2}\right)
$$

so that Eq. (126) is satisfied. We can now apply the dynamic scaling I\&I design method so long as the following assumptions are satisfied.

Assumption 1 (A1). There exists a function $\boldsymbol{\mu}_{2}: D \subset \mathbb{R}^{2 n} \rightarrow \mathbb{R}^{p}$ such that Eq. (127) holds, where $D$ is a compact set, and $\Psi\left(\mathbf{x}_{1}, \mathbf{x}_{2}\right) \in \mathbb{R}^{n \times p}$ is smooth and locally Lipschitz for any $\left(\mathbf{x}_{1}, \mathbf{x}_{2}\right)$ in $D$. Thus, if $\varpi_{1} \in \mathbb{R}^{2 n}$ and $\varpi_{2} \in \mathbb{R}^{2 n}$ are bounded then there is a positive constant $L_{\Psi}$ such that

$$
\left\|\Psi\left(\varpi_{1}\right)-\Psi\left(\varpi_{2}\right)\right\|_{2} \leq L_{\Psi}\left\|\varpi_{1}-\varpi_{2}\right\|_{2}
$$


Assumption 2 (A2). The smallest eigenvalue of $M\left(\mathbf{x}_{1}\right)$ is uniformly lower bounded and the bound denoted as $M_{m}$ is known.

Remark 6. Since $\Phi_{2}\left(\mathbf{x}_{1}, \mathbf{x}_{2}\right)$ is, in general, smooth and locally Lipschitz for all $\left(\mathbf{x}_{1}, \mathbf{x}_{2}\right) \in \mathbb{R}^{2 n}$, the choice $\Psi\left(\mathbf{x}_{1}, \mathbf{x}_{2}\right)=-\Phi_{2}\left(\mathbf{x}_{1}, \mathbf{x}_{2}\right)$ is always feasible. Note that only filter-free controllers require this local Lipschitz condition for implementation. Moreover, since mass matrices are uniformly bounded for any robot systems whose joints are all revolute as mentioned in Property 2, our assumptions are not very restrictive. It is also important to point out that introducing the functions $\boldsymbol{\mu}_{1}$, $\boldsymbol{\mu}_{3}$ and $\boldsymbol{\mu}_{2}$ that satisfy Eqs. (117), (125) and (127) gives a general way to construct $\boldsymbol{\beta}$ as mentioned in Remark 1.

The goal is to remove the effect of $\Psi\left(\mathbf{x}_{1}, \mathbf{x}_{2}\right)$ in parameter estimation by adopting a dynamic scaling factor. By the CE principle, we first propose our control law as

$$
\mathbf{u}=-\Phi\left(\mathbf{y}, \mathbf{x}_{1}, \mathbf{x}_{2}\right)(\hat{\boldsymbol{\theta}}+\boldsymbol{\beta})
$$

and then design an update law in a similar way suggested in the previous section. The details of the final expression for the dynamic scaled adaptive controller are presented in appendix, which are omitted here in the interest of brevity.

\section{Numerical Simulations}

\section{A. Attitude Tracking Control}

Consider the rigid-body attitude dynamics (1), where the inertia matrix is given by [9]

$$
J=\left[\begin{array}{ccc}
20 & 1.2 & 0.9 \\
1.2 & 17 & 1.4 \\
0.9 & 1.4 & 15
\end{array}\right]
$$

with its smallest eigenvalue bound $J_{m}=14.2672$. The body attitude is initially given by $\mathbf{q}_{v}(0)=$ $(0.1826,0.1826,0.1826)$ and $q_{0}(0)=\sqrt{1-\mathbf{q}_{v}^{T}(0) \mathbf{q}_{v}(0)}$ with zero rate. The initial reference quaternion is set to $\mathbf{q}_{r}(0)=(1,0,0,0)$ and the corresponding reference rate profile is given by $\omega_{r}(t)=$ 
$\left(\omega_{r}(t), \omega_{r}(t), \omega_{r}(t)\right)$, where

$$
\omega_{r}(t)=0.3\left(1-e^{-0.01 t^{2}}\right) \cos t+t e^{-0.01 t^{2}}(0.08 \pi+0.006 \sin t) .
$$

The initial parameter estimates are chosen such that $\tilde{\boldsymbol{\theta}}(0)=(1,1,1,1,1,1)$. We simulate the closedloop system using $\eta_{3}(0)=1$ and the other design parameters:

$$
k_{p}=k=0.7, k_{r}=0.2, k_{1}=k_{2}=k_{3}=1, \Gamma=50 I_{3}, v=0.5, \epsilon=0.00001
$$

for the filter-free case and

$$
k_{p}=k_{v}=0.7, k_{r}=0.2, k=k_{H}=0.01, k_{1}=k_{2}=k_{3}=1, \Gamma=50 I_{3}, v=0.5, \epsilon=0.00001
$$

for the filter case. The tracking errors for both cases converge to zero with good transient performances shown in Fig. 1. Even though there are some differences in $\eta_{1}(t)$ and $\eta_{2}(t)$ as seen in Fig. 2, the control effort and the convergence properties of the parameter estimate errors are almost identical as illustrated in Fig. 3. Also, the time histories of the error signals and control effort are very similar to those in [9], which shows outperformance of non-CE controllers. To see the effect

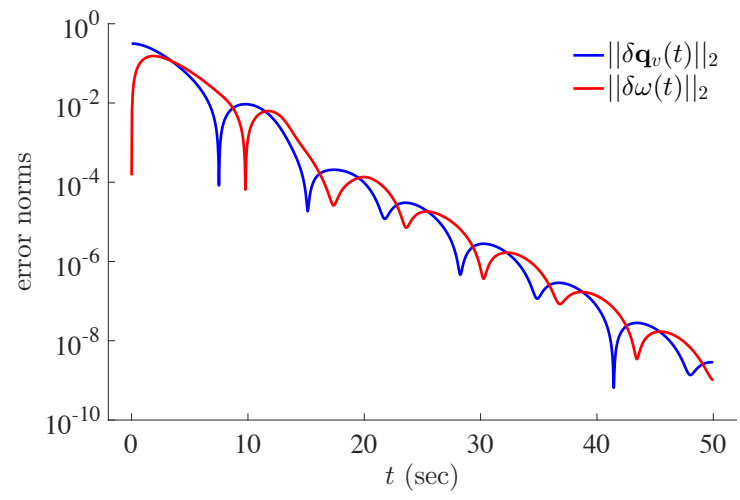

a) Error norms (Non-filter)

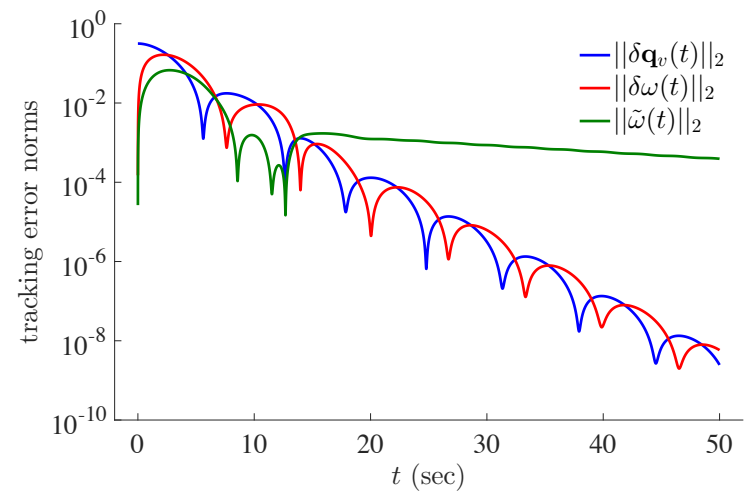

b) Error norms (Filter)

Figure 1. Time histories of $\left\|\delta \mathbf{q}_{v}(t)\right\|_{2},\|\delta \omega(t)\|_{2}$ and $\|\tilde{\boldsymbol{\omega}}(t)\|_{2}$.

of the initial rate tracking error $\delta \omega(0)$, we set the initial rate as $\omega(t)=(1,1,1)$ and simulate the closed-loop system using the same reference trajectories. To ensure $\eta_{2}(t)<1$ for all $t \geq 0$, we only 


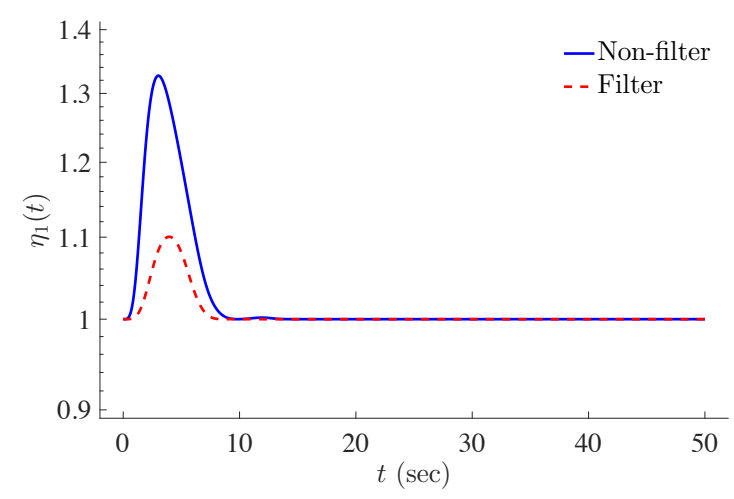

a) $\eta_{1}(t)$

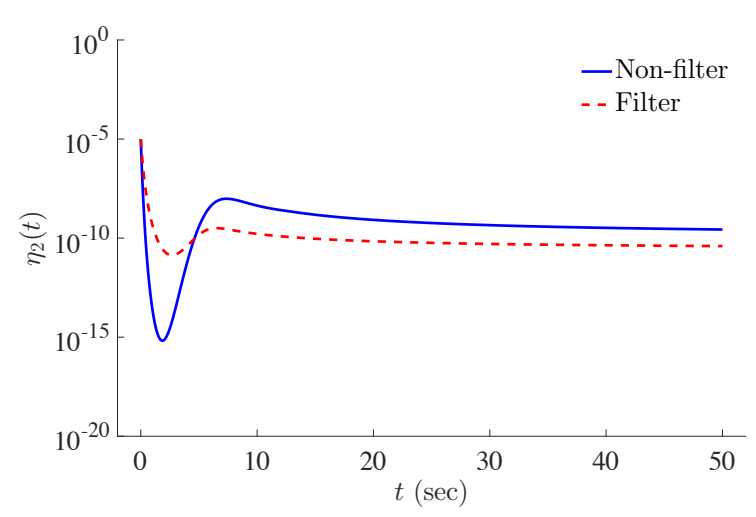

b) $\eta_{2}(t)$

Figure 2. Time histories of $\eta_{1}(t)$ and $\eta_{2}(t)$

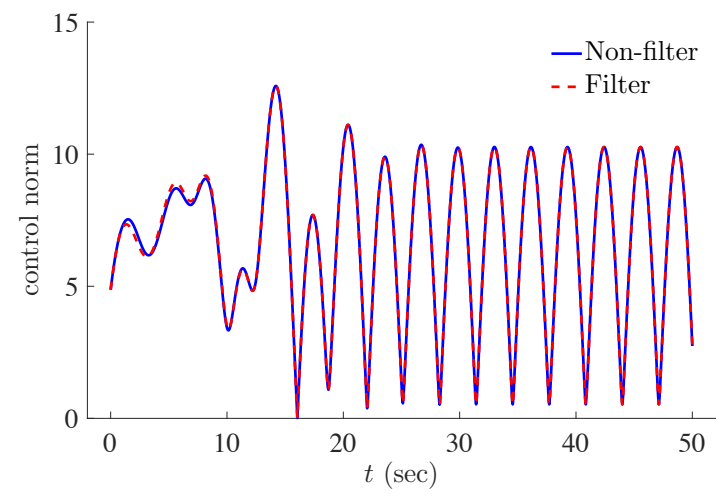

a) Control norm

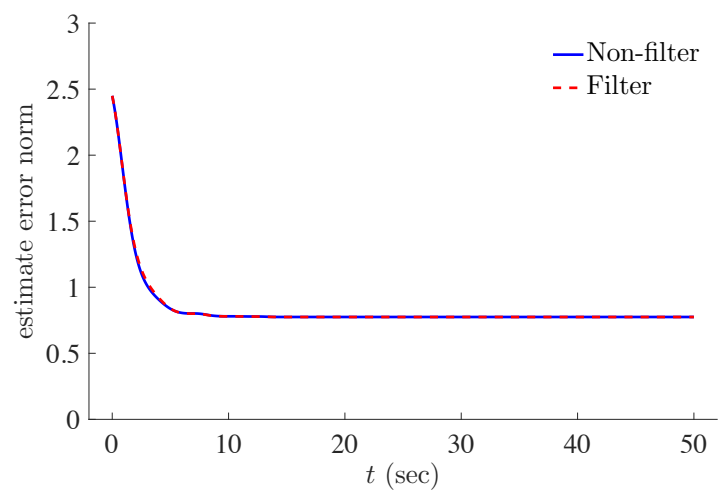

b) Estimate error norm

Figure 3. Time histories of $\|\mathbf{u}(t)\|_{2}$ and $\|\tilde{\boldsymbol{\theta}}(t)\|_{2}$

change the value of $k_{2}$ to 70 for the filter-free controller. In Fig. 4, the filter-free controller shows a faster convergence in the parameter estimation, but relatively large control effort is applied in the transient region. Thus, when the control power is limited or initial tracking errors are not small enough, the filter method would be desirable.

\section{B. 2-Link Planar Robot Tracking Control}

We consider the same dynamic model appearing in the recent filter-based I\&I results [10]. The parameters for the proposed controller are selected such that tracking performance is similar to that from the reference.

We consider the 2-link planar robot arm as depicted in Fig.5. The inertia and centripetal- 


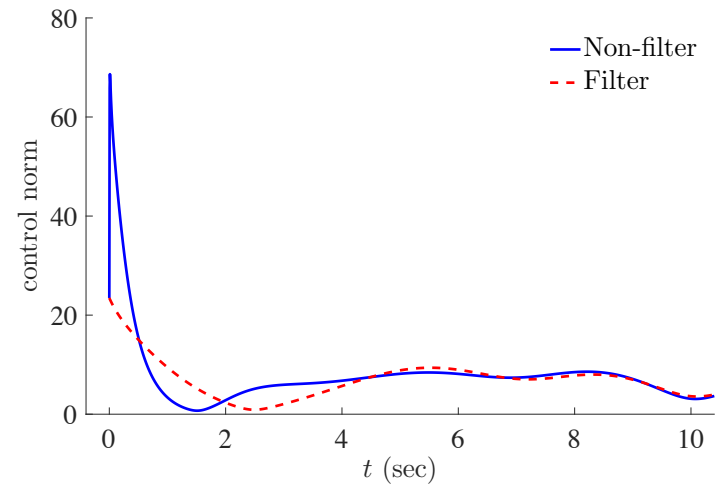

a) Control norm

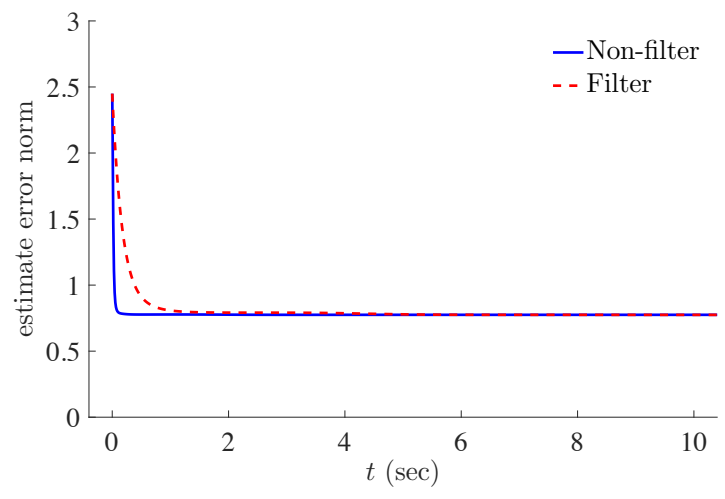

b) Estimate error norm

Figure 4. Time histories of $\|\mathbf{u}(t)\|_{2}$ and $\|\tilde{\boldsymbol{\theta}}(t)\|_{2}$ with large $\delta \boldsymbol{\omega}(0)$

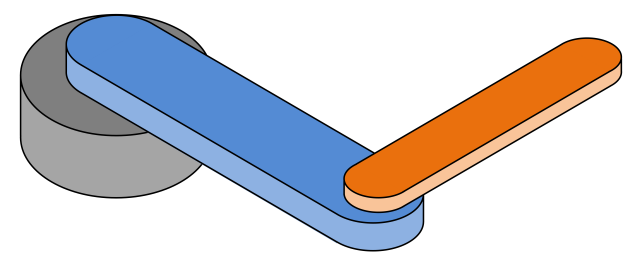

a) 2-link planar manipulator

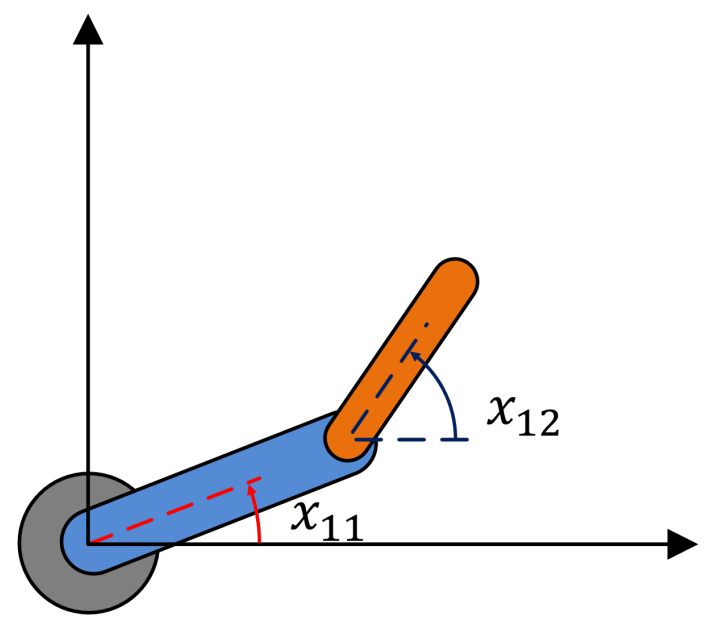

b) Generalized coordinates

Figure 5. Example of 2-link planar robot manipulator.

Coriolis matrices are given by

$$
M\left(\mathbf{x}_{1}\right)=\left[\begin{array}{cc}
\theta_{1}+2 \theta_{3} \cos x_{12} & \theta_{2}+\theta_{3} \cos x_{12} \\
\theta_{2}+\theta_{3} \cos x_{12} & \theta_{2}
\end{array}\right]
$$

and

$$
C\left(\mathbf{x}_{1}, \mathbf{x}_{2}\right)=\left[\begin{array}{cc}
-\theta_{3} x_{22} \sin x_{12} & -\theta_{3}\left(x_{21}+x_{22}\right) \sin x_{12} \\
\theta_{3} x_{21} \sin x_{12} & 0
\end{array}\right],
$$

where $\mathbf{x}_{1}=\left(x_{11}, x_{12}\right) \in \mathbb{R}^{2}$ and $\mathbf{x}_{2}=\left(x_{21}, x_{22}\right) \in \mathbb{R}^{2}$ are the states and $\boldsymbol{\theta}^{*}=\left(\theta_{1}, \theta_{2}, \theta_{3}\right) \in \mathbb{R}^{3}$ is the system parameter vector. The effect of gravity and friction is neglected and the true values for the 
parameters are set to $\boldsymbol{\theta}^{*}=(3.6,0.2,0.15)$. The lower bound of the smallest eigenvalue of $M\left(\mathbf{x}_{1}\right)$ is known to be $M_{m}=0.1672$ and the following $\boldsymbol{\mu}_{2}\left(\mathbf{x}_{1}, \mathbf{x}_{2}\right)$ is chosen such that A1 is satisfied:

$$
\boldsymbol{\mu}_{2}\left(\mathbf{x}_{1}, \mathbf{x}_{2}\right)=\left[\begin{array}{c}
0 \\
0 \\
x_{21} x_{22}\left(x_{21}+x_{22}\right) \sin x_{12}
\end{array}\right]
$$

The corresponding $\Psi\left(\mathbf{x}_{1}, \mathbf{x}_{2}\right)$ is then given by

$$
\Psi\left(\mathbf{x}_{1}, \mathbf{x}_{2}\right)=\left[\begin{array}{ccc}
0 & 0 & 0 \\
0 & 0 & 2 x_{21}\left(x_{21}+x_{22}\right) \sin x_{12}
\end{array}\right] .
$$

When a filter for $\mathbf{x}_{2}$ is adopted for the filter method, we use

$$
\left\|H\left(\mathbf{x}_{1}, \mathbf{x}_{2}, \hat{\mathbf{x}}_{2}\right)\right\|_{2}^{2}=4 \sin ^{2} x_{12}\left[\left(x_{21}+x_{22}+\hat{x}_{21}\right)^{2}+\hat{x}_{21}^{2}\right],
$$

where $\hat{\mathbf{x}}=\left(\hat{x}_{21}, \hat{x}_{22}\right) \in \mathbb{R}^{2}$ is the filter state. The initial conditions for the states $\mathbf{x}_{1}(0)$ and $\mathbf{x}_{2}(0)$ are set to zero, and the reference position is given by $\mathbf{x}_{r 1}(t)=(\cos t+2, \sin t+2)$. The parameter estimate vector is initially set to $\hat{\boldsymbol{\theta}}(0)=(2.6,0,0)$ and the controller parameters are selected as

$$
k_{p}=k=1, k_{r}=2, k_{1}=0.01, k_{2}=4, k_{3}=0.2, \Gamma=7.5 I_{3}, v=0.5, \epsilon=0.00001
$$

for the filter-free case and

$k_{p}=k v=1, k=1, k_{H}=0.1, k_{r}=2, k_{1}=0.01, k_{2}=4, k_{3}=0.2, \Gamma=7.5 I_{3}, v=0.5, \epsilon=0.00001$

for the filter case.

The results are shown in Figs. 6, 7 and 8. The tracking errors converge to zero with an acceptable transient. Also the parameter estimates converge to the true values due to the persistently exciting reference signals. Since $k_{2} \eta_{3}^{2}(t)$ dominates over $\|\tilde{\Psi}(t)\|_{2}^{2}, \eta_{2}(t)$ approaches close to zero as the tracking errors converge to zero as illustrated in Fig. 7b. As a result, the dynamic gain returns 


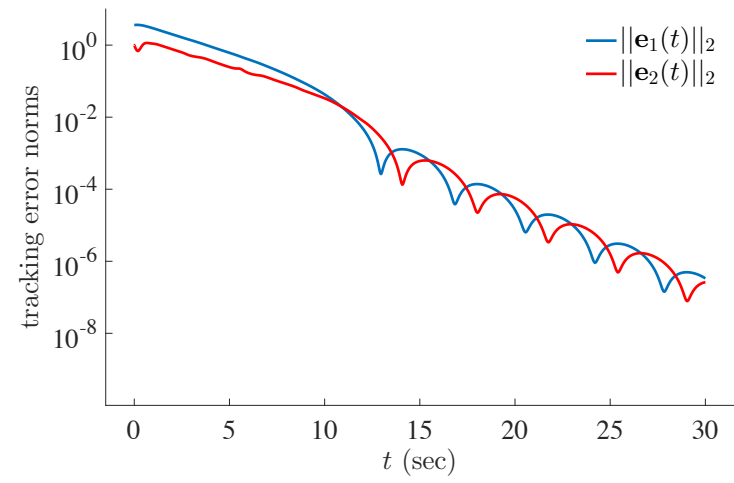

a) Error norms (Non-filter)

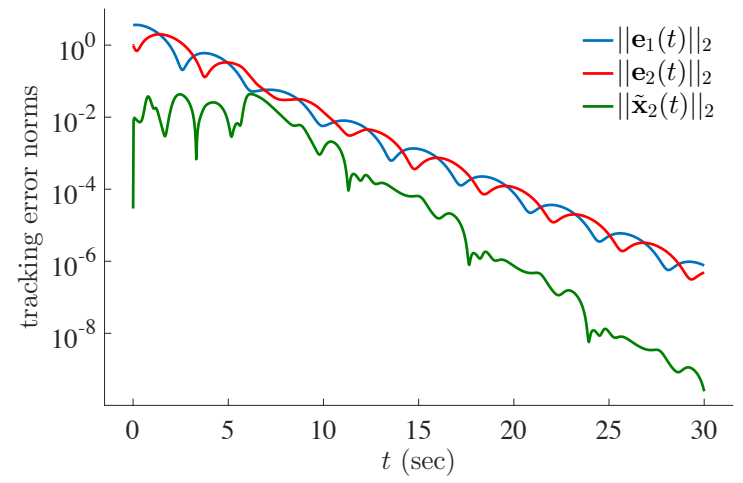

b) Error norms (Filter)

Figure 6. Time histories of $\left\|\delta \mathbf{q}_{v}(t)\right\|_{2},\|\delta \omega(t)\|_{2}$ and $\|\tilde{\boldsymbol{\omega}}(t)\|_{2}$.

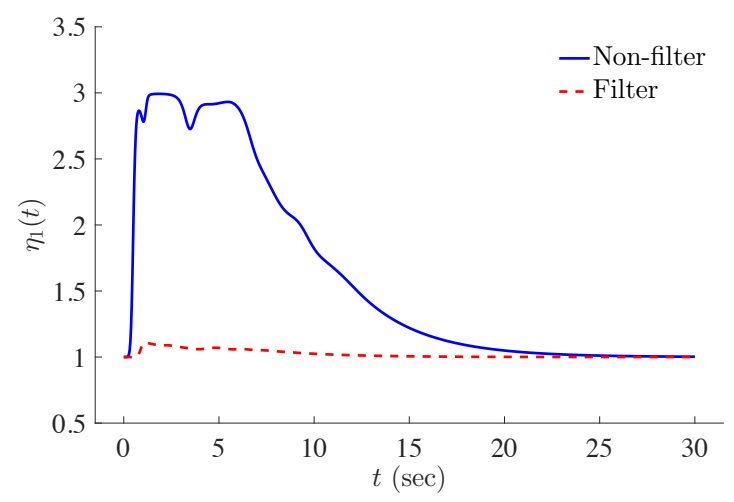

a) $\eta_{1}(t)$

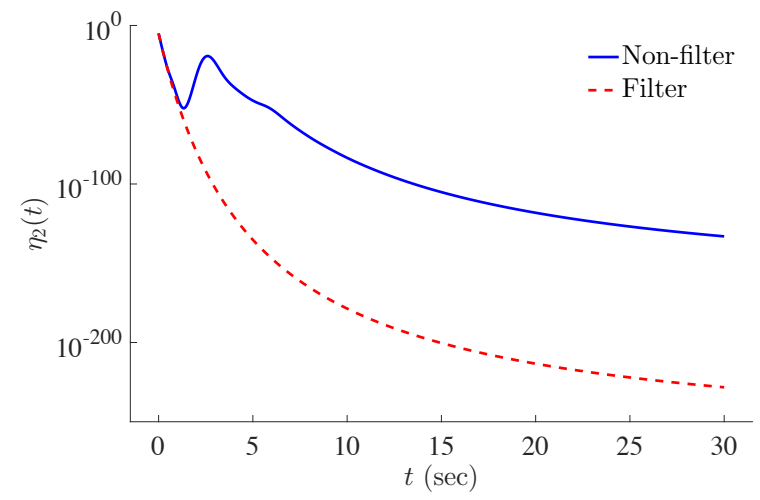

b) $\eta_{2}(t)$

Figure 7. Time histories of $\eta_{1}(t)$ and $\eta_{2}(t)$

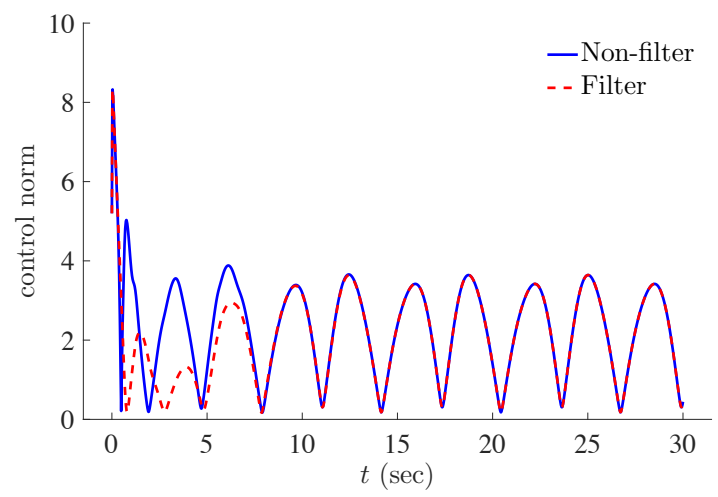

a) Control norm

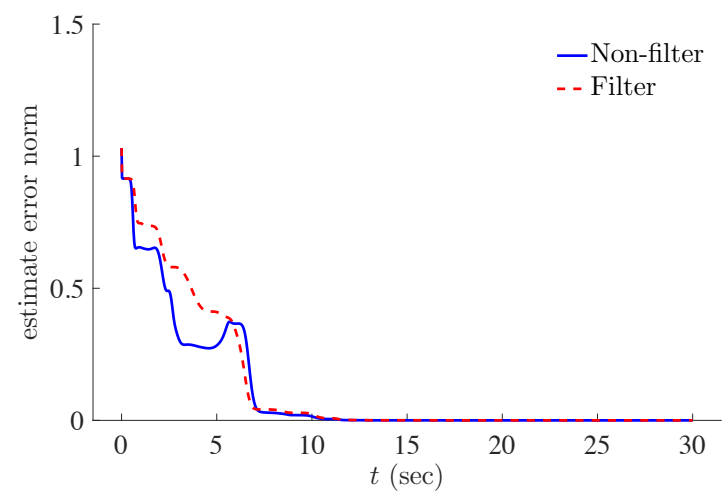

b) Estimate error norm

Figure 8. Time histories of $\|\mathbf{u}(t)\|_{2}$ and $\|\tilde{\boldsymbol{\theta}}(t)\|_{2}$ 
close to the initial value. It is observed that $\eta_{1}(t)$ is approximately upper bounded by $(v+1) / v=3$ for both controllers.

\section{Conclusion}

Novel dynamically scaled Immersion and Invariance (I\&I) adaptive controllers are proposed for the rigid-body attitude and the $n$-link robot manipulator control problems. For each problem, we assume that the inertia parameters are unknown but a lower bound of the smallest eigenvalue of the inertia matrix is available. A dynamic scaling factor is employed to circumvent the integrability obstacle typically arising in I\&I adaptive control design. Based on where the dynamic scaling factor appears, both filter-free and filter-dependent controllers are designed. Notably, by introducing three scalar dynamics, the monotonically increasing scaling factor is eliminated from the closedloop states. In addition, we have more control knobs to adjust and tune the range of the dynamic gain that contains the scaling factor. Depending on the choices of design parameters, the dynamic gain is shown to be pulled down to the vicinity of the initial value as the tracking errors converge to zero. The performances of the controllers are also analyzed through numerical simulations. The spacecraft and 2-link planar manipulator are considered. Particularly, for the attitude control case, the simulation results of the filter-free and filter-dependent controllers are compared when the initial tracking error of the rate state is intentionally chosen to be a large value. Further research will focus on removing the need of the inertia matrix bound or applying a projection mechanism to the partially known inertia parameters. To find better ways to restrict behavior of the dynamic scaling factor would be a challenging problem.

\section{Appendix: Summary of I\&I Estimators for the Robot Manipulator Problem}

Suppose that A1 and A2 are satisfied, and that appropriate $\boldsymbol{\mu}_{1}, \boldsymbol{\mu}_{2}$ and $\boldsymbol{\mu}_{3}$ are obtained so that we can define

$$
\boldsymbol{\mu}\left(k_{v}, \overline{\mathbf{y}}, \mathbf{x}_{1}, \mathbf{x}_{2}\right)=\boldsymbol{\mu}_{1}\left(k_{v}, \overline{\mathbf{y}}, \mathbf{x}_{1}, \mathbf{x}_{2}\right)+\boldsymbol{\mu}_{2}\left(\mathbf{x}_{1}, \mathbf{x}_{2}\right)+\boldsymbol{\mu}_{3}\left(\mathbf{x}_{1}, \mathbf{x}_{2}\right) .
$$

For any $\boldsymbol{\xi} \in \mathbb{R}^{n}$, we also define

$$
\tilde{\boldsymbol{\mu}}\left(k_{v}, \overline{\mathbf{y}}, \mathbf{x}_{1}, \mathbf{x}_{2}, \boldsymbol{\xi}\right)=\boldsymbol{\mu}\left(k_{v}, \overline{\mathbf{y}}, \mathbf{x}_{1}, \mathbf{x}_{2}\right)-\boldsymbol{\mu}\left(k_{v}, \overline{\mathbf{y}}, \mathbf{x}_{1}, \boldsymbol{\xi}\right),
$$




$$
\tilde{\Psi}\left(\mathbf{x}_{1}, \mathbf{x}_{2}, \boldsymbol{\xi}\right)=\Psi\left(\mathbf{x}_{1}, \mathbf{x}_{2}\right)-\Psi\left(\mathbf{x}_{1}, \boldsymbol{\xi}\right)
$$

and

$$
\boldsymbol{\mu}^{\prime}=\dot{\tilde{\boldsymbol{\mu}}}-\frac{\partial \boldsymbol{\mu}\left(k_{v}, \overline{\mathbf{y}}, \mathbf{x}_{1}, \mathbf{x}_{2}\right)}{\partial \mathbf{x}_{2}} \dot{\mathbf{x}}_{2}
$$

Since $\Psi\left(\mathbf{x}_{1}, \mathbf{x}_{2}\right)$ is assumed to be smooth, there exists a function $H\left(\mathbf{x}_{1}, \mathbf{x}_{2}, \boldsymbol{\xi}\right)$ such that

$$
\left\|\tilde{\Psi}\left(\mathbf{x}_{1}, \mathbf{x}_{2}, \boldsymbol{\xi}\right)\right\|_{2} \leq\left\|H\left(\mathbf{x}_{1}, \mathbf{x}_{2}, \boldsymbol{\xi}\right)\right\|_{2}\left\|\mathbf{x}_{2}-\boldsymbol{\xi}\right\|_{2}
$$

In the I\&I estimator design, $\boldsymbol{\xi}=\mathbf{x}_{r 2}$ for the filter-free estimator and $\boldsymbol{\xi}=\hat{\mathbf{x}}_{2}$ for the filter-dependent estimator. For the dynamic gains, we use the same dynamic extension defined in Eqs. (79), (80) and (81) with $k_{r}>1 /\left(4 M_{m}\right)$.

\section{A. Filter-Free Estimator}

The update law is proposed as

$$
\dot{\hat{\boldsymbol{\theta}}}=-\Gamma\left[\boldsymbol{\mu}^{\prime}+\Psi^{T}\left(\mathbf{x}_{1}, \mathbf{x}_{r 2}\right) \mathbf{f}-\dot{\Psi}^{T}\left(\mathbf{x}_{1}, \mathbf{x}_{r 2}\right) \mathbf{e}_{2}+\left(\Phi^{T}\left(\mathbf{y}, \mathbf{x}_{1}, \mathbf{x}_{2}\right)+\tilde{\Psi}^{T}\left(\mathbf{x}_{1}, \mathbf{x}_{2}, \mathbf{x}_{r 2}\right)\right)\left(\mathbf{f}-k_{p} \mathbf{e}_{1}-k_{\nu} \mathbf{e}_{2}\right)\right]
$$

and

$$
\boldsymbol{\beta}=\Gamma\left(\tilde{\boldsymbol{\mu}}\left(k_{v}, \overline{\mathbf{y}}, \mathbf{x}_{1}, \mathbf{x}_{2}, \mathbf{x}_{r 2}\right)-\Psi^{T}\left(\mathbf{x}_{1}, \mathbf{x}_{r 2}\right) \mathbf{e}_{2}\right),
$$

where $\Gamma=\Gamma^{T}>0$ and $k_{v}=k \eta_{1}$ with $k>0$.

\section{B. Filter-Dependent Controller}

Let $\tilde{\mathbf{x}}_{2}=\mathbf{x}_{2}-\hat{\mathbf{x}}_{2}$. Then the update law is proposed as

$$
\dot{\hat{\boldsymbol{\theta}}}=-\Gamma\left[\boldsymbol{\mu}^{\prime}+\Psi^{T}\left(\mathbf{x}_{1}, \hat{\mathbf{x}}_{2}\right) \dot{\hat{\mathbf{x}}}_{2}-\dot{\Psi}^{T}\left(\mathbf{x}_{1}, \hat{\mathbf{x}}_{2}\right) \tilde{\mathbf{x}}_{2}+\left(\Phi^{T}\left(\mathbf{y}, \mathbf{x}_{1}, \mathbf{x}_{2}\right)+\tilde{\Psi}^{T}\left(\mathbf{x}_{1}, \mathbf{x}_{2}, \hat{\mathbf{x}}_{2}\right)\right)\left(\mathbf{f}-k_{p} \mathbf{e}_{1}-k_{v} \mathbf{e}_{2}\right)\right]
$$


and

$$
\boldsymbol{\beta}=\Gamma\left(\tilde{\boldsymbol{\mu}}\left(k_{v}, \overline{\mathbf{y}}, \mathbf{x}_{1}, \mathbf{x}_{2}, \hat{\mathbf{x}}_{2}\right)-\Psi^{T}\left(\mathbf{x}_{1}, \hat{\mathbf{x}}_{2}\right) \tilde{\mathbf{x}}_{2}\right)
$$

where $\Gamma=\Gamma^{T}>0$ and $k_{v}>0$. The corresponding filter is given by

$$
\dot{\hat{\mathbf{x}}}_{2}=k_{f} \tilde{\mathbf{x}}_{2}-k_{p} \mathbf{e}_{1}-k_{\nu} \mathbf{e}_{2}+\mathbf{f},
$$

where $k_{f}$ is a dynamic gain defined as

$$
k_{f}=\left(k+k_{H}\left\|H\left(\mathbf{x}_{1}, \mathbf{x}_{2}, \hat{\mathbf{x}}_{2}\right)\right\|_{2}^{2}\right) \eta_{1}
$$

with $k, k_{H}>0$.

\section{Acknowledgment}

The results reported in this work were supported in part by National Aeronautics and Space Administrations Johnson Space Center through Grant Number NNX14AK46A (Technical Manager: Dr. Christopher N. D'Souza).

\section{References}

[1] Åström, K. J., "Theory and applications of adaptive control - a survey," Automatica, Vol. 19, No. 5, 1983, pp. 471-486.

doi: 10.1016/0005-1098(83)90002-X

[2] Narendra, K. S. and Annaswamy, A. M., Stable adaptive systems, Courier Corporation, 2012.

[3] Astolfi, A. and Ortega, R., "Immersion and invariance: a new tool for stabilization and adaptive control of nonlinear systems," Automatic Control, IEEE Transactions on, Vol. 48, No. 4, 2003, pp. 590-606.

doi: 10.1109/TAC.2003.809820

[4] Astolfi, A., Karagiannis, D., and Ortega, R., Nonlinear and adaptive control with applications, Springer-Verlag, London, 2008. 
[5] Ortega, R., Hsu, L., and Astolfi, A., "Immersion and invariance adaptive control of linear multivariable systems," Systems \& control letters, Vol. 49, No. 1, 2003, pp. 37-47. doi: 10.1016/S0167-6911(02)00341-9

[6] Astolfi, A., Hsu, L., Netto, M. S., and Ortega, R., "Two solutions to the adaptive visual servoing problem," Robotics and Automation, IEEE Transactions on, Vol. 18, No. 3, 2002, pp. 387-392.

doi: 10.1109/TRA.2002.1019475

[7] Karagiannis, D., Astolfi, A., and Ortega, R., "Two results for adaptive output feedback stabilization of nonlinear systems," Automatica, Vol. 39, No. 5, 2003, pp. 857-866.

doi: 10.1016/S0005-1098(03)00003-7

[8] Zachi, A. R., Hsu, L., Ortega, R., and Lizarralde, F., "Dynamic control of uncertain manipulators through immersion and invariance adaptive visual servoing," The International Journal of Robotics Research, Vol. 25, No. 11, 2006, pp. 1149-1159.

doi: $10.1177 / 0278364906072039$

[9] Seo, D. and Akella, M. R., "High-performance spacecraft adaptive attitude-tracking control through attracting-manifold design," Journal of Guidance, Control, and Dynamics, Vol. 31, No. 4, 2008, pp. 884-891.

doi: $10.2514 / 1.33308$

[10] Seo, D. and Akella, M. R., "Non-certainty equivalent adaptive control for robot manipulator systems," Systems \& Control Letters, Vol. 58, No. 4, 2009, pp. 304-308.

doi: 10.1016/j.sysconle.2008.11.008

[11] Lee, K. W. and Singh, S. N., "Multi-input noncertainty-equivalent adaptive control of an aeroelastic system," Journal of Guidance, Control, and Dynamics, Vol. 33, No. 5, 2010, pp. 1451-1460.

doi: $10.2514 / 1.48302$ 
[12] Karagiannis, D., Sassano, M., and Astolfi, A., "Dynamic scaling and observer design with application to adaptive control," Automatica, Vol. 45, No. 12, 2009, pp. 2883-2889. doi: 10.1016/j.automatica.2009.09.013

[13] Sonneveldt, L., van Oort, E., Chu, Q., and Mulder, J., "Immersion and invariance based nonlinear adaptive flight control," AIAA Guidance, Navigation and Control Conference, Toronto, Ontario, Canada, 2010, pp. 1-18.

doi: $10.2514 / 6.2010-7690$

[14] Ji, Y., Zong, Q., and Zeng, F., "Immersion and invariance based nonlinear adaptive control of hypersonic vehicles," Control and Decision Conference (CCDC), 2012 24th Chinese, IEEE, 2012, pp. 2025-2030.

doi: 10.1109/CCDC.2012.6244326

[15] Liu, X., Ortega, R., Su, H., and Chu, J., "On adaptive control of nonlinearly parameterized nonlinear systems: Towards a constructive procedure," Systems \& Control Letters, Vol. 60, No. 1, 2011, pp. 36-43.

doi: 10.1016/j.sysconle.2010.10.004

[16] Junkins, J. L., Akella, M. R., and Robinett, R. D., "Nonlinear adaptive control of spacecraft maneuvers," Journal of Guidance, Control, and Dynamics, Vol. 20, No. 6, 1997, pp. 1104 1110.

doi: $10.2514 / 2.4192$

[17] Schaub, H., Akella, M. R., and Junkins, J. L., "Adaptive control of nonlinear attitude motions realizing linear closed loop dynamics," Journal of Guidance, Control, and Dynamics, Vol. 24, No. 1, 2001, pp. 95-100.

doi: $10.2514 / 2.4680$

[18] Costic, B., Dawson, D., De Queiroz, M., and Kapila, V., "Quaternion-based adaptive attitude tracking controller without velocity measurements," Journal of Guidance, Control, and Dy- 
namics, Vol. 24, No. 6, 2001, pp. 1214-1222.

doi: $10.2514 / 2.4837$

[19] Slotine, J.-J. E. and Li, W., "On the adaptive control of robot manipulators," The international journal of robotics research, Vol. 6, No. 3, 1987, pp. 49-59.

doi: $10.1177 / 027836498700600303$

[20] Ortega, R. and Spong, M. W., "Adaptive motion control of rigid robots: A tutorial," Automatica, Vol. 25, No. 6, 1989, pp. 877-888.

doi: 10.1016/0005-1098(89)90054-X

[21] Sadegh, N. and Horowitz, R., "Stability and robustness analysis of a class of adaptive controllers for robotic manipulators," The International Journal of Robotics Research, Vol. 9, No. 3, 1990, pp. 74-92.

doi: 10.1177/027836499000900305

[22] Yang, S., Hashemi, E. K., and Akella, M. R., "Dynamic Scaling Based Adaptive Controllers for Aeroelastic Systems," 17th Yale Workshop on Adaptive and Learning Systems, 2015.

[23] Yang, S. and Akella, M. R., "Adaptive Control Boosted through Dynamically Adjusted Feedback Gains: Applications to Controlling Euler-Lagrange Systems," Society of Engineering Science 52nd Annual Technical Meeting, 2015.

[24] Yang, S. and Akella, M. R., "Dynamically Scaled Immersion and Invariance Approach for Spacecraft Attitude Tracking Control," 26th AAS/AIAA Space Flight Mechanics Meeting, 2016, AAS 16-352.

[25] Schaub, H. and Junkins, J. L., Analytical mechanics of space systems, AIAA Education Series, AIAA, Reston, VA, 2003.

[26] Mazenc, F. and Akella, M. R., "Quaternion-based stabilization of attitude dynamics subject to pointwise delay in the input," American Control Conference (ACC), 2014, IEEE, 2014, pp. 
4877-4882.

doi: 10.1109/ACC.2014.6858703

[27] Spong, M. W., Hutchinson, S., and Vidyasagar, M., Robot modeling and control, Vol. 3, Wiley New York, 2006. 